Supporting Information for

\title{
Mining of sucrose synthases from Glycyrrhiza uralensis and their application in the construction of an efficient UDP- recycling system
}

Liang Zhang, Yanan Gao, Xiaofei Liu, Fang Guo, Congxuan Ma, Jianhua Liang, Xudong Feng ${ }^{*}$, Chun Li

Institute for Synthetic Biosystem/Department of Biochemical Engineering, School of Chemistry and Chemical Engineering, Beijing Institute of Technology, Beijing 100081, China

*Correspondence to Xudong Feng (xd.feng@bit.edu.cn) 
Table S1. Primers used for PCR amplification of sucrose synthase genes from Glycyrrhiza uralensis (The primers used for cloning GuSUS1 and GuSUS2 was highlighted with underline).

\begin{tabular}{|c|c|}
\hline SUS-F1 & TGGGTCGCGGATCCGAATTCATGGCTTCTACATCAAYTT \\
\hline SUS-R1 & GGTGCTCGAGTGCGGCCGCACTGCTCCTGTGTGCATAGAT \\
\hline SUS-F2 & TGGGTCGCGGATCCGAATTCATGTCTCAACCTAAGCTTG \\
\hline SUS-R2 & GGTGCTCGAGTGCGGCCGCAATTTGCATCATCMTCCTTTG \\
\hline SUS-F3 & TGGGTCGCGGATCCGAATTCATGAAGGATCGCCACGTAC \\
\hline SUS-R3 & GGTGCTCGAGTGCGGCCGCAATTTGCATCATCCTTTGCTA \\
\hline SUS-F4 & TGGGTCGCGGATCCGAATTCATGAAGGATCGCCACGTAC \\
\hline SUS-R4 & GGTGCTCGAGTGCGGCCGCACCTTTCATAAATTCTTTGAA \\
\hline SUS-F5 & TGGGTCGCGGATCCGAATTCATGGCTTCTACACCAGGCT \\
\hline SUS-R5 & GGTGCTCGAGTGCGGCCGCAACTTTGCAATCTGGACTTTG \\
\hline SUS-F6 & TGGGTCGCGGATCCGAATTCATGGCTGTGAACGAATGGC \\
\hline SUS-R6 & GGTGCTCGAGTGCGGCCGCAGAATTGTTGAATATCACAGT \\
\hline$\frac{\text { SUS-F7 }}{\text { (GuSus1) }}$ & TGGGTCGCGGATCCGAATTCATGGCTACCGATCGTTTGA \\
\hline$\frac{\text { SUS-R7 }}{(\text { GuSus1) }}$ & GGTGCTCGAGTGCGGCCGCACTCCTCAACAGCTAGGGGCA \\
\hline SUS-F8 & TGGGTCGCGGATCCGAATTCATGGCTTCTGTACCAGCCT \\
\hline SUS-R8 & GGTGCTCGAGTGCGGCCGCAAGCTCCAAGTAACCTTCGCA \\
\hline SUS-F9 & TGGGTCGCGGATCCGAATTCATGGCTGGGAATGAGTGGA \\
\hline SUS-R9 & GGTGCTCGAGTGCGGCCGCAGATTCCTCCAGATTTTGAAA \\
\hline SUS-F10 & TGGGTCGCGGATCCGAATTCATGGCTGGGAATGAATGGA \\
\hline SUS-R10 & GGTGCTCGAGTGCGGCCGCACATTAGCCCAGATCGAGATA \\
\hline SUS-F11 & TGGGTCGCGGATCCGAATTCATGCCATCTTCAAGGTTCG \\
\hline SUS-R11 & GGTGCTCGAGTGCGGCCGCACAAGTCCCGGAATTTGAGAA \\
\hline$\frac{\text { SUS-F12 }}{\text { (GuSus2) }}$ & TGGGTCGCGGATCCGAATTCATGGCTAATGATCATCTRA \\
\hline$\frac{\text { SUS-R12 }}{\text { (GuSus2) }}$ & GGTGCTCGAGTGCGGCCGCACTCTTCAATAGCAAGGGGCA \\
\hline
\end{tabular}


Table S2. Primers used for PCR amplification of GuSUS1, GuSUS2 from Glycyrrhiza uralensis and their mutants for heterologous expression in E. coli.

\begin{tabular}{ll}
\hline GuSus1-F & AGCAAATGGGTCGCGGATCCTAAATGGCTACCGATCGTTTGACC \\
GuSus1-R & TGGTGGTGGTGGTGCTCGAGCTCCTCAACAGCTAGGGGCA \\
GuSus1- $\Delta 9-\mathrm{F}$ & AGCAAATGGGTCGCGGATCCTAAATGCACAGTCTCCGTGAGAGGC \\
GuSus1- $\Delta 9-\mathrm{R}$ & TGGTGGTGGTGGTGCTCGAGCTCCTCAACAGCTAGGGGCA \\
GuSus2-F & AGCAAATGGGTCGCGGATCCTAA $\underline{\text { ATGGCTAATGATCATCTAAC }}$ \\
GuSus2-R & TGGTGGTGGTGGTGCTCGAGCTCTTCAATAGCAAGGGGCA \\
GuSus2- $\Delta 9-\mathrm{F}$ & AGCAAATGGGTCGCGGATCCTAAATGCACTCTTTCCGCGAGAGGCT \\
GuSus2- $\Delta 9-\mathrm{R}$ & TGGTGGTGGTGGTGCTCGAGCTCTTCAATAGCAAGGGGCA \\
\hline
\end{tabular}


Table S3. List of 12 putative SUSs amino acid sequence of $G$. uralensis

$>$ Glyur000124s00011937.1

MAGNDWLNSYLEAILDVGPGLDDAKSSLLLRERGRFSPTRYFVEEVIGFDETDLHRSW VRASSSRSPQERNTRLENMCWRIWNLARQKKQLESETLQRVNRRRLERERGRREATAD MSEDLSEGEKGDPVSDVSAHGGDSGRARLPRISSADAMEMWAKSQKGKKLYIVLISIH GLIRGENMELGRDSDTGGQVKYVVELARALGSMPGVYRVDLLTRQVSAPGVDWSYG EPTEMLSPRNTDEFGDDMGESSGAYIIRIPFGPRNKYIPKENLWPYISEFVDGALSHIIQM SKALGEQIGSGHAVWPVAIHGHYADAGDSAALLSGALNVPMLFTGHSLGRDKLEQLLK QGRLSRDEINTTYKIMRRIEAEELTLDGSEIVITSTRQEIEEQWRLYDGFDPVLERKLRAR IRRNVSCYGRFMPRMAIIPPGMEFHHIVPLDGDIEGELEGNFDHPAPQDPPIWSEIMRFFT NPRKPMILALARPDPKKNITTLVKAFGECRPLRELANLTLIMGNRDGIDEMSSTNASVLL SVLKLIDKYDLXGQVAYPKHHKQSDVPDIYRLAAKTKGVFINPAFIEPFGLTLIEAAAYG LPMVATKNGGPVDIHRVLDNGLLVDPHDQQSIADALLKLVSNKQLWAKCRQNGLKNIH LFSWPEHCKNYLSKIATCKPRHPQWQRSEDGGESSESESPGDSLRDIQDLSLNLKFSLDG ERSGDSGNDNSLDPDGNAADRSARLENAVLSWSKGISKDIRRGGAAEKSDQNSNAGKF PPLRRRNHLFVIAVDCDTISDLCETTKAIFESVGKERAEGSVGFILSTSLTISEIQSFLISGG LSPSDFDAYICNSGSDLYYPSLNPEDRPFVGDLYYHSHIEYRWGGEGLRKTLIRWASSITE KKGENDEQIVSPSEQLSTDYCYTFKVRKPGMAPPVKELRKLVRIQALRCHPIYCQNGTR LNVIPVLASRSQALRYLYVRWGFELSKMVVFVGECGDTDYEGLVGGLHKSVILKGVGS SAISQLHNNRNYPLSDVMPLDSPNIVEATEGSSXADIQALLEKVGYLTG

$>$ Glyur000202s00015414.1

MSQPKLGRLPSIRDRVEGTLSAHRNELVSLLSRYVEQGKGILQPHNLIDELDNIKGQDQ ASGDLKNGPFGDIIRSAQEAIVLPPFVAIAVRPRPGIWEHVRVNVFELSVEQLSVSEYLRF KEELVDGTNIDPCVLELDFEPFNASFPRPTRSSSIGNGVQFLNRHLSSIMFRNKDSLDPLL DFLRAHKYKGHGLMLNDRIHSVSKLQSALAKAEEYLSKLASDTPYSEFEYVLQGMGFE RGWGDTAERVLEMMHLLLELLQAPDPSTLETFLGRVPMVFNVVILSPHGYFGQANVLG LPDTGGQVVYILDQVRALENEMLLRIKKQGLDFTPRILVVTRLIPDAKGTTCNQRLEKV SGTEHTHILRVPFRSDKGILNKWISRFDVWPYLETYAKDVASEIAAELQGHPDFIIGNYS DGNLVASLLACKMGVTQCTIAHALEKTKYPDSGTYWKKFDDKYHFSCQFTADLIAMN SADFIITSTYQEIAGTKNTVGQYESHTAFTLPGLYRVVHGIDVFDPKFNIVSPGADMCIYF PYFEKQKRLTSLHGSIEKLLYDPEQTDEYIGTLKDKSKPIIFSMARLDRVKNITGLVESFG KNSKLRELVNLVVVAGYIDVSKSRDREEIAEIGKMYDLIKTHNLDGDFRWIAAQTNRAR NGELYRYIADTKGAFVQPAFYEAFGLTVVESMTCGLPTFATCHGGPAEIIEHGKSGFHID PYHPDQASELLVEFFQKCKDDPGHWNKISDGGLXRIYERFTWRIYSERLMTLAGVYSF WKYVSKLERRETRRYLEMFYTLKYRDLAKSVPLAKXDDAN

$>$ Glyur000307s00023022.1

MASTPGLKRTESMTDHNMADASKQSRYHKRRCFAKYLEXGRRIIKLHHLMEEMEQVI NDKSDRNQILEGNLGFLLSYTQEAVVDPPYVAFAVRSDPGVWEFVRVSSENLSVEPLTST DYLKFKERIYDEKWYVVYILDQVRALEEELLLKIKQQGLSVKPQILVVTRLIPDAQGTK CNQELESINGTKHSNILRVPFLTDKGILRQWVSRFDIYPYLERFTQDATTKILNLMEGNP DLIIGNYTDGNLVASLMASKLRITQGTIAHALEKTKYEDSDVKWKELDPKYHFSCQFM ADTIAMNASDFIISSTYQEIAGSKDKPGQYESHATFTLPGLCRVVSGINIFDPKFNIAAPG 
ADQSVYFPYTEKDKRLTQFHPAIEDLLYSKGDNKEHIGYLENRRKPIIFSMARLDVVKN LTGLVEWYGKNQRLRSLVNLVIVGGFFDPLKSKDREEMGEIRKMHDLIGKYQLKGQXR WIAAQTDRHRNGELYRFIADTKGAFVQPALYEAFGLTVIEAMNCGLPTFATDHGGPAEII VDGISGFHIDPLNGEESSNKIADFFEKCKVDSIHWNVISAAGLQRINECYTWKIYANKLL NMGSIYTFWRQVNNKQKEAKQRYIRMFYNLXFKNLVKTIPIPSDEPQQPVSKQQSLKK QGTSRRSKSRLQS

>Glyur000386s00015334.1

MAVNEWLNGYLEAILDVGSSSMRSKQRNDGKLKMTKFEKYRDHQREETLFNPTKYFV EEVVNSFDESDLYRTWAKVIATRNNSRERGNRLENMCWRIWHLTRKKKQLSEGEKEK GDANVVSELCVKDNNNISRINSEMQLCIHGLVRGENMELGRDSDTGGQVKYVVELAR ALANTKGIYRVDLLTRQIASPEVDSIYGEPIEMYIPKESLWPHIPEFVDGALNHIVNMAR ALGEQVNGGKPTWPYVIHGHYADAGEIAAHLSGALSVPMVLTGHSLGRNKFEQLLKQ GRLSREAINATYKIMRRIEAEELXVDAAEMVVTSTRQEIEEQWGLYDGFDLKXERKLR VRRRRGVIPPGMDFSYVKMRDSVEGEGDLKSLIGSDRAQRKRNLPPIWSEIMRFFTNPH KPMILALSRPDPKKNVMTLLKAFGECQALRELANLTLILGNRDDIEEMSDSSSTVLTMV LMLIDKYDLYGQVAYPKHHKQSEVPEIYRLAAKSKAAAYGLPVVATKNGGPVDILKAL NNGLLIDPHDQKAIADSLVKLVADKNLWLECRKKGLKNIHRFSWPEHCRNYLSHIEHCR NRHSTSRLEITPVAEEPISDSLRDVEDLSFRFSTEVDSKLDGEMDLATRQKEIIEAITRRVS STGNSNASYFPGRRQRLVVIAADCYDGDGNRTEALQAIIKNVMKGAGQGISAGRVGLV LLTGSSLQETIEALNSFQVNIEEFDALVCNTGSEMYYPWKDLIVDADYEAHVEYAWPGE NIRSMVTRLXRIEDGEEDDIVEEYASACSSRCYSYSVKPGAMIRKIDDLRQRLRMRGFR CNLVYTHAGLRLNVIPLFASRKQALRYLSVKWGIDLSKVVVFVGEKGDTDYEELVAGI QKTLVLRGVVENGSERLLRSEDNFKREDVFSQDSPNIIYAEKSYEDCDIQQF

>Glyur000519s00019093.1

MATDRLTRVHSLRERLDETLTANRNEILALLSRIEAKGKGILQHHQVIAEFEEIPEENRHK LMDGAFGEVLRSTQEAIVLPPWVALAVRPRPGVWEYLRVNVHALVVEELQPAEFLRFK EELVDGSSNGNFVLELDFEPFTASFPRPTLNXSIGNGVQFLNRHLSAKLFHDKESLHPLL EFLRLHSYKGKTLMLNDRIQTPDSLQHVLRKAEEYLGTLXPETPYSVFEHKFQEIGLER GWGDTAERVLESIQLLLDLLEAPDPCTLETFLGRIPMVFNVVILSPHGYFAQDNVLGYP DTGGQVVYILDQVRALENEMLHRIKQQGLDIVPRILIITRLLPDAVGTTCGQRLEKVFGT EHCHILRVPFRNEKGMVRKWISRFEVWPYLETYTEDVAHELAKELQGKPDLIVGNYSD GNIVASLLAHKLGVTQCTIAHALEKTKYPESDIYWKKFEEKYHFSCQFTADLFAMNHT DFIITSTFQEIAGSKDTVGQYESHTAFTLPGLYRVVHGIDVFDPKFNIVSPGADQTIYFPY TDTSRRLTSFHPEIEELLYSSVENEEHICVLKDRNKPIIFTMARLDRVKNITGLVEWYGKN AKLRELVNLVVVAGDRRKESKDLEEKAEMKKMYGLIETYKLNGQFRWISSQMNRVRN GELYRVICDTKGAFVQPAVYEAFGLTVVEAMTCGLPTFATCNGGPAEIIVHGKSGFHIDP YHGAAAADLLVEFFEKCKADPSHWDNISHGGLQRIEEKYTWQIYSERLLTLTGVYGFW KHVSNLDRRESRRYLEMFYALKYRKLAESVPLAVEE

$>$ Glyur000693s00025759.1

MASVPALKRTDSIVDNMPDALRQSRYHMKRCFAKYLEKGRRIMKIHHLMEEMDRTID DKSERNQVLEGTLGFILSTTQEAVVDPPYVAFAIRPNPGVWEYVXVNSEDLYVEAISPTD 
YLKFKERVHDEKWAIDENAFEADFGAFDIGIPQLTLSSSIGKGLQFVSKFLTSRYSGKLA KTQPIVDYLLSLNHQGESLMINDSLSSAAKLQMALVVADVFLSALPKDTAYQNIELRLK EWGFEKGWGDTAGRVKESMGTLSEVLQAPDHENLEKFFSRVPTIFKVVVFSVHGYFGQ SDVLGLPDTGGQALEAELLLRIKEQGLNVKPQILVVTRLIPDALGTRCHQEMEPINDTK YSHILRVPFQTEKGILRRWDATTKILDLMDGKPDLVIGNYTDGNLVASLMSRKLGITQGT IAHALEKTKYEDSDVKWKELDPKYHFSCQFMADTVAMNASDFIITSTYQEIAGSKDRP GQYESHAAFTLPGLCRVVSGINVFDPKFNIAAPGADQSIYFPYTEKDKRLSQFHPAIEDL LFNKVDNNEHIGYLAERRKPIIFSMARLDVVKNLTGLVEWYAKNRRLRNLVNLVIVGGF FDPSKSKDREEMAEIKKMYDLIEKYQLKGQFRWIAAQTNRYRNGELYRCIADTRGAFV QPALYEAFGLTVIEAMNCGLPTFATNQGGPAEIIVDGVSGFHIDPLNGDESSNKIADFFEK CKVDPSQWSVISAAGLRRINECYTWKIYANKLVNMGNIYTFWRLVNKEQKEAKQRYIH MFYNLIFKNLVKTVPLPSGEPQKPVGKQSSLKQQGSSIRRSQSTLRRLLGA

$>$ Glyur000723s00020864.1

MAGNEWINGYLEAILSTGSATTVEEHQRAVAPTPRESVHFNPTKYFVEEVVSSVDESDL HRTWLKVVATRNTRERSSRLENMCWRIWHLARKKKQLEVEEHHRLAYRRWEREQGR RDATEDMSEDLSEGEKGDIIGELIQMETPKKKLQGQISNLEVWSDDKKEKKIYIVLVRD SDTGGQIKYVVELARALAKMPGVYRVDLFTRQISSPEVDWSYGEPTEMLTAGTDEDDD DTGESSGAYIIRIPFGPRDKYLQKELLWPHVQEFVDGALAHILNMSKVLGEQVGGGQPV WPYVIHGHYADAGDSAALLSGALNVPMVLTGHSLGRNKLEQLIKQGRQSREDINSTYK IMRRIEAEELSLDAAELVITSTRQEIDEQWGLYDGFDVKLEKVLRARARRGVNCHGRY MPRMAVIPPGMDFSNVVPQEDGPEVDGELSQLTAGADGSSPKALPAIWXEVMRFFTNP HKPMILALSRPDPKKNITTLLKAFGECRPLRKLANLTLIMGNRDDIDEMSSGNASVLTT VLKLIDKYDLYGQVAYPKHHKQYDVPEIYRFAAKTRGVFINPALVEPFGLTLIEAAAHGL PLVATKNGGPVDINRALNNGLLVDPHDQQAIADALLKLLSEKNLWHECRKNGWKNIHL FSWPEHCRTYLTRVAACRIRHPQWQTNTPEDDVAVEESFNDSLKDEQDMSLRLSIDGDL AAASGGLEVQDQVKRLLSKIRKSDSGSNQDGGSKVKKPDSGSNSENMLLDNVTNKYP LLRRRRRLIVIALDSYDNNGAPDKKITEIVQRIIKAVQLDPQTARVSGFALSTAMPMQET VEFLTSGNIPVNEFDALICSSGSEVYYPGIYTEDGKLLPDPDYEVHMDYRWGCEGLKKT IWKLMNTSEGVENPEKTSSPIEEDLKSSNAHCISYKIKDLSKARKVDDLRQKLRMRGLR CHPMYLFVRWRMNVANMYVILGETGDTDYEEMISGTHKTIIMKGVASKGSEELLRGPG SYQRDXIVPNESPLVTYISETTEEKIANALKQLSKSGGI

$>$ Glyur000805s00021382.1

MAGNEWINGYLEAILSTGASTIEEQKPAAVTLGDGGHYNPTKYFVEEVVTSVDESDLYR TWIKVVATRNTRERSSRLENMCWRIWHLARKKKQLEWEEGQRLANRRWEREQGRRD ATEDMSEDLSEGEKGDSXGEMVQCDTPRKRFQRQVSNLEVWSDDKKENKLYIVLISM HGLVRAENMELGRDSDTGGQIKYVVELARALAKMPGVYRVDLFTRQISSPEVDWSYG EPTEMLTAGADDDDSIGESSGAYIIRIPFGPRXKYLQKELLWPYIQEFVDGALTHILNMSK VLGEQVGGGQPVWPYVIHGHYADAGDSAAILSGALNVPMVLTGHSLGRNKLEQLLKQ GRQSKEDINSTYKMMRRIEAEELSLDAAELVITSTKQEIEEQWGLYDGFDVKVEKVLRA RARRGVNCHGRYMPRMAVIPPGMDFSNVVNQEELPEADGELGQLTGGVEGSSPKSMP AIWSEVMRFFTNPHXPVILALSRPDPKKNLTTLLKAFGESRPLRELANLMLIMGNRDDI DEMSSGNASVLTTVLKLIDKYDLYGQVAYPKHHKQSDVPEIYRFAGKTKGVFINPALVE 
PFGLTLIEAAAHGLPMVATKNGGPVDIHRALNNGLLVDPHDQQAITNALIKLLSEKNLW HECRKNGWKNIHLFSWPEHCRTYLTRVAACRMRHPQWQTNTPGDDITVEESFNDSLKD VADMSLRLSIDADLAAASAGPEMQDQVKRVLSKMKKSDSVGLSDTDGGNKLPDNVTG KYPLLRRRRRLIVIAVDFYDKNGAPDKKMIQIVQRIIKAVXLDPQTARVSGFALSTAMPM LETIEFLKSCNIQVNDFXALICSSGSQLYYPGAYTEDGKLLPDPDYAAHIDYRWGCEGLK KTIWNLMNIAEGGEKYSSPIEEDLKSSNAHCISYKIKDLSKAKRVDDLRQKLRMRGLRC HPMYCRGLSYMQVIPLLASRAQALRYLFVRWRLNVANMYVILGETGDTDYEELISGTH KTIIMKGVVSKGSEEXLRGPGSYQRADVVPDKSPLVAYISETTEXKIANALKELSRSGLM

$>$ Glyur001102s00033853.1

MAVFRLPSVTVPSPSQPMPSRHLSPQHSLLRRFNNSFPPLLRQISTTTPPTTLSSTTSFFSK GPRSSXSVNVAVRSQLRYPMVSPNDHWGIWSTLFATGAFGLWSEKTKIGSMVSGALVST LVGLAASNLGILPHDAPAYSVVLEFLLPLTIPLLLFGANLHQVVRSTGTLLLAFLLGSVAT IVGTLVAFLLVPMRSLGPDNWKIAAALMAVNYVAISEALGMSASVLAAGVAADNVICA LYFVVLFALASKIPAEAAIPTTAATYLTKLTGIQGGTLPGVTAVIVILATLLPKQIGPLVPAG HTVALVLMQVFFVVVGASGSIWNVIRTAPSIFMFALVQVTIHLLLVLGLGKLFKLDLKLL LLASNANIGGPTTACGMAKAKGWESLVVPGILTGIFGVAIATFLGIGFGMELYSNNITGKI PDELGNLTRGSCCSSFPPYVAFVVRPNPGFWEFVKVNADDLQVDGIEASEYLKYKEMV FDEKW

$>$ Glyur001223s00024742.1

MPSSRFDRASSLXDRVQDTLSSYRNELISLLSRYVAKGKGILQPHDLLDEVEKIVKEDEG MQKLKDSPFVKALESAKEAIVLPPFVSIAFRPRPGVWEYVRVNVFELIVDNLSVAEYLR FKXELVDGECTDNYVLELDFEPFNATFPRPNRSSSIGNGVHFLNRHLSSFMFRNKESLDP LLAFLRTHRYDGHAMMLNDRIRSISKLQSSLTKAEEFLSKLLPDTPYSEFEYELQGMGFE RGWGDTAQRVLEMVHLLLDIFQAPDPNTLEAFLGRIPMVFNVAIVSPHGYFGQANVLG LPDTGGQVVYILDQVRALENEMLLRIRKQGLDVSPKILIVTRLIPDAKGTCNQRLERVS GTGHTYILRVPFRTKNGILRKWISRFDVWPYLETFAEDASNEIAAELQGVPDLIIGNYSD GNLVATLLSYKLGITQCNIAHALEKTKYPDSDIYWKKYEDKYHFTCQFTADLISMNNAD FIITSTYQEIAGSKNNVGQYESHTAFTLPGLYRVVHGIDVFDPKFNIVSPGADMCIYFPYS EREKRLTSLHGSIEKMVYGAEQNEEHIGLLNDRSKPIIFSMATLDQVKNMTGLVECFGK SSKLRELVNLVVVGGYIDVQKSIDREEIGEIEKMHGLIEQYNLHGQFRWIKAQMNRARN VVEAMTCGLPTFATCHGGPXEIIEHGISGFHIDPHHPDQVAAILINFFEQCQHDPEYWNKI SDAGLRRIHERYTWKVYSERLLTLAGVYGFWKHVSKLERRETRRYLEMFYILKFRDL

$>$ Glyur001836s00028590.1

MITTMMIADTVAVNAAKSLGLLRGYQFPIADSGIDQISAMIQTCDAVAPPPVKPGKYRVS RRTLLRRKRRTKRRLSGDESGNEGLFFGDGGDGPFSGGSGGFGGGGGGSWNSNRFGD GHNWDEPSSSLPDPAFDFVYQVLSWIMLSNCLHFAIKKIVRIIADGSIXDSDREKVPARL APIC

>Glyur001957s00039090.1

MANDHLTHSHSFRERLDETLTGHRNEILALLSRIEAKGKGILQHHQVIAEFEEIPEENRQ KLLNGAFGEVLRSTQEAIVLPPFVALAVRPRPGVWEYLRINVHGLVVDELRAAEYLKFK 
EELVEGSSNGNFVLELDFEPFNASFPRPTLNKSIGNGVEFLNRHLSAKLFHGKESMKPLL EFLRLHSYNGKTMMLNDRIQNLNSLQHVLRKAEEYLSTIAPQTPYSEFEHEFQEIGLER GWGDTAERVLEMIQLLLDLLEAPDPCTLETFLGRIPMVFNVVILSPHGYFAQDNVLGYP DTGGQVVYILDQVRALENEMLNRIKKQGLDITPRILIITRLLPDAVGTTCGQRLEKVYNT EHCHILRVPFRTEKGIVRKWISRFEVWPYLETYTEDVANELAKELQGKPDLIVGNYSDG NIVASLLAHKLGVTQCTIAHALEKTKYPESDIYWKKFEDKYHFSCQFTADLFAMNHTDF IITSTFQEIAGSKDTVGQYESHTAFTLPGLYRVVHGIDVFDPKFNIVSPGADMSIYFPYTE TKRRLTSFHPEIEELLYSSVENEEHICVLKDRNKPIIFTMARLDRVKNITGLVEWYGKNA KLRELVNLVVVAGDRRKESKDLEEKAEMKKMYGLIETYKLNGQFRWISSQMNRVRNG ELYRVICDTKGAFVQPAVYEAFGLTVVEAMTCGLPTFATCNGGPAEIIVHGKSGYHIDPY HGDRAAELLVEFFEKSKADPSHWDKISHGGLKRIHEKYTWQIYSERLLTLTGVYGFWK HVSNLDRRESKRYLEMFYALKYRKLAESVPLAIEE 
Table S4. The nucleotide sequences of GuSUS1 and GuSUS2

$>$ GuSUS1

ATGGCTACCGATCGTTTGACCCGTGTTCACAGTCTCCGTGAGAGGCTCGATGAAACC TTGACTGCTAATAGAAATGAAATTTTGGCCCTTCTCTCAAGGATCGAAGCCAAGGGC AAGGGGATCCTGCAACACCACCAGGTCATTGCTGAGTTTGAGGAAATTCCTGAGGA GAATAGACATAAGCTGATGGATGGGGCATTTGGAGAAGTCTTGAGATCCACACAGG AAGCCATAGTTTTACCACCATGGGTTGCTCTGGCTGTTCGTCCAAGGCCTGGTGTTT GGGAGTACCTGAGAGTGAATGTGCACGCTCTTGTTGTCGAAGAGTTGCAACCTGCT GAGTTTCTCCGCTTCAAGGAGGAACTTGTTGATGGAAGTTCTAATGGCAACTTTGTG CTTGAGTTGGACTTTGAACCATTTACTGCATCCTTCCCCCGCCCAACTCTCAACAAG TCAATTGGAAATGGTGTGCAATTCCTCAACCGTCACCTTTCTGCAAAACTCTTCCAT GACAAGGAGAGCTTGCATCCACTTCTGGAATTCCTCAGACTTCACAGCTACAAGGG AAAGACATTGATGTTGAATGACAGAATTCAAACCCCGGATTCTCTTCAACATGTTCT GAGGAAAGCTGAAGAGTATCTTGGAACACTTTCTCCTGAGACACCCTACTCAGTATT TGAGCACAAGTTCCAGGAGATCGGTTTGGAGAGAGGGTGGGGTGACACCGCGGAG CGTGTCCTCGAGTCCATCCAACTCCTCTTGGATCTTCTTGAGGCTCCTGACCCTTGCA CCCTTGAGACTTTCCTTGGAAGGATCCCCATGGTCTTTAATGTTGTGATCCTTTCGCC CCACGGTTACTTTGCCCAAGATAATGTCTTGGGATACCCTGATACCGGTGGCCAGGTT GTTTACATCTTGGATCAAGTTCGCGCCTTGGAGAATGAGATGCTCCATCGCATTAAGC AACAAGGCTTGGATATCGTCCCTCGCATTCTCATTATCACCCGTCTTCTCCCCGATGC AGTAGGAACTACCTGTGGCCAACGACTCGAGAAGGTCTTTGGAACCGAGCATTGCC ACATTCTTCGAGTTCCCTTCAGAAACGAGAAGGGAATGGTTCGCAAGTGGATCTCA AGATTCGAAGTCTGGCCATACCTAGAAACTTACACTGAGGATGTTGCCCATGAACTT GCCAAAGAGTTGCAAGGCAAGCCAGATCTGATTGTTGGAAACTACAGTGATGGAAA CATTGTTGCCTCTTTGTTGGCACATAAATTAGGTGTCACTCAGTGTACCATTGCTCAT GCACTTGAGAAGACCAAGTACCCTGAATCTGACATTTACTGGAAAAAATTCGAAGA GAAATATCACTTCTCTTGCCAATTCACAGCTGATCTCTTTGCTATGAACCACACAGAC TTCATCATCACCAGTACCTTCCAAGAGATTGCTGGAAGCAAGGACACTGTTGGACA GTATGAGAGTCACACTGCCTTCACCCTTCCTGGACTCTACCGTGTCGTGCACGGTAT TGATGTCTTTGATCCAAAATTCAACATTGTATCTCCCGGAGCTGATCAGACCATCTAC TTCCCCTACACCGACACCAGCCGCAGGCTGACATCCTTCCACCCCGAAATCGAAGA GCTTCTTTACAGCTCAGTGGAGAATGAAGAGCACATATGTGTATTGAAGGACCGCAA CAAGCCAATTATCTTCACCATGGCGAGGTTGGACCGTGTGAAGAACATCACTGGACT TGTCGAGTGGTACGGCAAGAACGCCAAGCTCCGTGAGCTGGTGAACCTTGTGGTTG TTGCCGGAGACAGGAGGAAGGAGTCCAAGGACTTGGAAGAGAAGGCCGAGATGAA GAAGATGTACGGCCTGATTGAGACCTACAAGCTGAATGGCCAATTCAGGTGGATCTC CTCTCAGATGAACCGGGTGAGGAACGGGGAGCTGTACCGTGTCATCTGCGACACAA AGGGAGCTTTCGTGCAGCCTGCTGTCTATGAGGCCTTTGGATTGACAGTTGTTGAGG CCATGACTTGTGGGTTGCCAACATTTGCAACATGCAATGGTGGCCCTGCTGAGATCA TTGTTCATGGCAAGTCTGGTTTCCACATTGACCCTTACCACGGCGCGGCCGCCGCCG ATCTCCTTGTTGAATTCTTTGAGAAGTGCAAGGCTGACCCATCTCACTGGGACAACA TCTCCCATGGTGGTCTCCAACGTATTGAAGAGAAGTATACATGGCAAATTTACTCTGA GAGGCTTCTCACTCTCACTGGTGTCTATGGCTTCTGGAAGCATGTGTCTAACCTTGA CCGCCGCGAGAGCCGCCGTTATCTTGAGATGTTCTATGCTCTCAAGTACCGCAAATT 
\begin{tabular}{l}
\hline GGCTGAGTCTGTGCCCCTAGCTGTTGAGGAG \\
\hline GuSUS2
\end{tabular}

ATGGCTAATGATCATCTAACCCATTCTCACTCTTTCCGCGAGAGGCTTGATGAAACTC TCACTGGTCACAGGAATGAGATTTTGGCCCTTCTATCAAGGATTGAAGCCAAGGGCA AGGGAATCCTGCAACACCACCAGGTCATTGCAGAGTTTGAAGAAATCCCTGAGGAG AATAGGCAAAAACTACTTAATGGGGCATTTGGAGAAGTTTTGAGATCTACCCAGGAA GCCATAGTGTTGCCATCATTTGTTGCTTTGGCTGTTCGTCCAAGGCCTGGTGTTTGGG AGTATCTTCGAATCAATGTTCACGGGCTTGTTGTTGATGAGCTTCGTGCTGCTGAGTA CCTGAAGTTCAAGGAGGAGCTTGTTGAAGGAAGTTCTAATGGCAACTTTGTGCTTG AGTTGGACTTTGAACCATTCAATGCTTCCTTCCCCCGACCAACTCTGAACAAGTCGA TCGGAAATGGCGTGGAGTTCCTCAACCGCCATCTTTCTGCTAAGCTCTTCCATGGCA AAGAGAGCATGAAGCCACTGCTTGAATTCCTCAGGCTTCACAGCTATAATGGAAAG ACTATGATGTTGAATGACAGAATTCAAAACCTGAATTCTCTCCAACATGTTTTGAGA AAAGCAGAAGAGTATCTGAGCACAATTGCTCCCCAAACACCCTACTCGGAATTCGA GCACGAATTCCAGGAGATCGGTTTGGAGAGAGGATGGGGTGACACCGCGGAGCGT GTCCTCGAGATGATCCAGCTTCTGCTGGATCTTCTCGAGGCACCTGACCCTTGCACC CTTGAGACATTCCTTGGAAGAATCCCTATGGTCTTTAATGTTGTCATCCTTTCTCCCC ATGGTTACTTTGCACAAGATAATGTCTTGGGATACCCTGATACCGGTGGACAGGTTGT GTACATCTTGGATCAAGTTCGTGCCTTGGAGAATGAGATGCTCAATCGTATCAAGAA ACAAGGCTTGGATATCACCCCTCGCATTCTCATTATCACTCGTCTTCTCCCTGATGCA GTGGGAACTACTTGTGGCCAGCGTCTTGAGAAGGTATATAACACTGAACATTGCCAC ATTCTTCGAGTTCCCTTTAGAACAGAGAAGGGAATTGTTCGCAAATGGATCTCAAGA TTTGAAGTCTGGCCCTACCTAGAGACTTACACTGAGGATGTTGCCAATGAACTTGCC AAAGAGCTTCAAGGCAAGCCTGATCTGATAGTTGGAAACTACAGTGATGGAAACAT TGTTGCCTCTTTGTTAGCACATAAATTGGGTGTGACTCAGTGTACCATTGCTCATGCA CTTGAGAAGACCAAGTATCCAGAGTCTGACATTTACTGGAAGAAATTTGAAGACAA ATACCACTTCTCATGCCAATTCACTGCTGATCTTTTTGCAATGAACCACACAGACTTT ATTATCACCAGCACCTTCCAAGAGATTGCTGGAAGCAAGGATACTGTTGGACAGTAT GAGAGTCACACTGCCTTCACCCTTCCGGGACTTTACCGCGTTGTTCACGGCATTGAT GTATTCGATCCGAAGTTCAACATTGTCTCTCCAGGAGCTGACATGAGCATTTACTTCC CATACACAGAAACCAAGCGTAGGTTGACATCATTCCACCCTGAGATTGAAGAACTTC TCTACAGCTCAGTGGAGAATGAGGAGCACATATGTGTATTGAAGGACCGGAACAAG CCAATCATCTTCACCATGGCAAGGTTGGACCGTGTGAAGAACATCACAGGACTTGTT GAGTGGTATGGCAAGAATGCCAAACTGAGGGAGTTGGTGAACCTTGTTGTTGTTGC TGGGGACAGGAGGAAGGTATCAAAGGACTTGGAAGAGAAAGCTGAGATGAAGAAA ATGTATGGACTCATTGAGACCTACAAGATGAATGGCCAATTCAGATGGATCTCTTCTC AGATGAATCGTGTGAGGAATGGAGAGCTCTACCGTGTCATTTGTGACACAAAGGGT GCATTCGTTCAGCCTGCTGTATATGAAGCCTTTGGTTTAACTGTTGTTGAGGCCATGA CTTGTGGGTTGCCAACATTTGCAACATGTAATGGTGGTCCTGCTGAGATCATTGTGC ATGGGAAATCTGGGTACCATATTGACCCTTACCATGGTGATCGTGCTGCTGAGCTTCT TGTTGAGTTCTTTGAGAAGAGCAAGGCTGATCCATCTCATTGGGACAAAATCTCCCA TGGAGGTCTCAAACGTATTCATGAGAAGTACACATGGCAAATTTACTCTGAGAGGCT CCTGACTCTCACTGGTGTCTATGGCTTCTGGAAGCATGTGTCAAACCTTGATCGCCG TGAGAGCAAACGCTACCTCGAGATGTTCTATGCCCTCAAGTACCGCAAATTGGCTGA 
Table S5. List of amino acid sequences in plant SUSs and bacterial SUSs with NCBI accession numbers

\begin{abstract}
$>$ GuSUS1 [Glycyrrhiza uralensis]
MATDRLTRVHSLRERLDETLTANRNEILALLSRIEAKGKGILQHHQVIAEFEEIPEENRHK LMDGAFGEVLRSTQEAIVLPPWVALAVRPRPGVWEYLRVNVHALVVEELQPAEFLRFK EELVDGSSNGNFVLELDFEPFTASFPRPTLNKSIGNGVQFLNRHLSAKLFHDKESLHPLL EFLRLHSYKGKTLMLNDRIQTPDSLQHVLRKAEEYLGTLSPETPYSVFEHKFQEIGLER GWGDTAERVLESIQLLLDLLEAPDPCTLETFLGRIPMVFNVVILSPHGYFAQDNVLGYP DTGGQVVYILDQVRALENEMLHRIKQQGLDIVPRILIITRLLPDAVGTTCGQRLEKVFGT EHCHILRVPFRNEKGMVRKWISRFEVWPYLETYTEDVAHELAKELQGKPDLIVGNYSD GNIVASLLAHKLGVTQCTIAHALEKTKYPESDIYWKKFEEKYHFSCQFTADLFAMNHT DFITTSTFQEIAGSKDTVGQYESHTAFTLPGLYRVVHGIDVFDPKFNIVSPGADQTIYFPY TDTSRRLTSFHPEIEELLYSSVENEEHICVLKDRNKPIIFTMARLDRVKNITGLVEWYGKN AKLRELVNLVVVAGDRRKESKDLEEKAEMKKMYGLIETYKLNGQFRWISSQMNRVRN GELYRVICDTKGAFVQPAVYEAFGLTVVEAMTCGLPTFATCNGGPAEIIVHGKSGFHIDP YHGAAAADLLVEFFEKCKADPSHWDNISHGGLQRIEEKYTWQIYSERLLTLTGVYGFW KHVSNLDRRESRRYLEMFYALKYRKLAESVPLAVEE
\end{abstract}

$>$ GuSUS2 [Glycyrrhiza uralensis]

MANDHLTHSHSFRERLDETLTGHRNEILALLSRIEAKGKGILQHHQVIAEFEEIPEENRQ KLLNGAFGEVLRSTQEAIVLPSFVALAVRPRPGVWEYLRINVHGLVVDELRAAEYLKFK EELVEGSSNGNFVLELDFEPFNASFPRPTLNKSIGNGVEFLNRHLSAKLFHGKESMKPLL EFLRLHSYNGKTMMLNDRIQNLNSLQHVLRKAEEYLSTIAPQTPYSEFEHEFQEIGLER GWGDTAERVLEMIQLLLDLLEAPDPCTLETFLGRIPMVFNVVILSPHGYFAQDNVLGYP DTGGQVVYILDQVRALENEMLNRIKKQGLDITPRILIITRLLPDAVGTTCGQRLEKVYNT EHCHILRVPFRTEKGIVRKWISRFEVWPYLETYTEDVANELAKELQGKPDLIVGNYSDG NIVASLLAHKLGVTQCTIAHALEKTKYPESDIYWKKFEDKYHFSCQFTADLFAMNHTDF IITSTFQEIAGSKDTVGQYESHTAFTLPGLYRVVHGIDVFDPKFNIVSPGADMSIYFPYTE TKRRLTSFHPEIEELLYSSVENEEHICVLKDRNKPIIFTMARLDRVKNITGLVEWYGKNA KLRELVNLVVVAGDRRKVSKDLEEKAEMKKMYGLIETYKMNGQFRWISSQMNRVRN GELYRVICDTKGAFVQPAVYEAFGLTVVEAMTCGLPTFATCNGGPAEIIVHGKSGYHIDP YHGDRAAELLVEFFEKSKADPSHWDKISHGGLKRIHEKYTWQIYSERLLTLTGVYGFW KHVSNLDRRESKRYLEMFYALKYRKLAESVPLAIEE

$>$ AED92894.1 SUS [Arabidopsis thaliana] MANAERMITRVHSQRERLNETLVSERNEVLALLSRVEAKGKGILQQNQIIAEFEALPEQ TRKKLEGGPFFDLLKSTQEAIVLPPWVALAVRPRPGVWEYLRVNLHALVVEELQPAEFL HFKEELVDGVKNGNFTLELDFEPFNASIPRPTLHKYIGNGVDFLNRHLSAKLFHDKESL LPLLKFLRLHSHQGKNLMLSEKIQNLNTLQHTLRKAEEYLAELKSETLYEEFEAKFEEIG LERGWGDNAERVLDMIRLLLDLLEAPDPCTLETFLGRVPMVFNVVILSPHGYFAQDNV LGYPDTGGQVVYILDQVRALEIEMLQRIKQQGLNIKPRILILTRLLPDAVGTTCGERLER VYDSEYCDILRVPFRTEKGIVRKWISRFEVWPYLETYTEDAAVELSKELNGKPDLIIGNY SDGNLVASLLAHKLGVTQCTIAHALEKTKYPDSDIYWKKLDDKYHFSCQFTADIFAMN HTDFIITSTFQEIAGSKETVGQYESHTAFTLPGLYRVVHGIDVFDPKFNIVSPGADMSIYF 
PYTEEKRRLTKFHSEIEELLYSDVENKEHLCVLKDKKKPILFTMARLDRVKNLSGLVEW YGKNTRLRELANLVVVGGDRRKESKDNEEKAEMKKMYDLIEEYKLNGQFRWISSQM DRVRNGELYRYICDTKGAFVQPALYEAFGLTVVEAMTCGLPTFATCKGGPAEIIVHGKS GFHIDPYHGDQAADTLADFFTKCKEDPSHWDEISKGGLQRIEEKYTWQIYSQRLLTLTG VYGFWKHVSNLDRLEARRYLEMFYALKYRPLAQAVPLAQDD

$>$ CAA26229.1 SUS [Zea mays]

MAAKLTRLHSLRERLGATFSSHPNELIALFSRYVHQGKGMLQRHQLLAEFDALFDSDK EKYAPFEDILRAAQEAIVLPPWVALAIRPRPGVWDYIRVNVSELAVEELSVSEYLAFKEQ LVDGQSNSNFVLELDFEPFNASFPRPSMSKSIGNGVQFLNRHLSSKLFQDKESLYPLLNF LKAHNYKGTTMMLNDRIQSLRGLQSSLRKAEEYLLSVPQDTPYSEFNHRFQELGLEKG WGDTAKRVLDTLHLLLDLLEAPDPANLEKFLGTIPMMFNVVILSPHGYFAQSNVLGYP DTGGQVVYILDQVRALENEMLLRIKQQGLDITPKILIVTRLLPDAAGTTCGQRLEKVIG TEHTDIIRVPFRNENGILRKWISRFDVWPYLETYTEDVSSEIMKEMQAKPDLIIGNYSDG NLVATLLAHKLGVTQCTIAHALEKTKYPNSDIYLDKFDSQYHFSCQFTADLIAMNHTDF IITSTFQEIAGSKDTVGQYESHIAFTLPGLYRVVHGIDVFDPKFNIVSPGADMSVYYPYTE TDKRLTAFHPEIEELIYSDVENSEHKFVLKDKKKPIIFSMARLDRVKNMTGLVEMYGKN ARLRELANLVIVAGDHGKESKDREEQAEFKKMYSLIDEYKLKGHIRWISAQMNRVRNG ELYRYICDTKGAFVQPAFYEAFGLTVIESMTCGLPTIATCHGGPAEIIVDGVSGLHIDPYH SDKAADILVNFFDKCKADPSYWDEISQGGLQRIYEKYTWKLYSERLMTLTGVYGFWK YVSNLERRETRRYIEMFYALKYRSLASQVPLSFD

>ABP88869.1 SUS [Medicago falcata]

MVRRLTRVHSLKERLDETLTANRNEILALLSRLEAKGKGILQHHRVIAEFEEIPEESRQKL TDGAFGEVLRSTQEAIVLPPWVALAVRPRPGIWEYLRVNVHALVVENLQPAEFLKFKEE LVDGSANGNFVLELDFEPFTASFPRPTLNKSIGNGVHFLNRHLSAKLFHDKESLHPLLEF LRLHSYKGKTLMLNDRIQNPDSLQHVLRKAEEYLSTIDPETPYSEFEHRFQEIGLERGW GDTAERVLESIQLLLDLLEAPDPCTLESFLDRIPMVFNVVILSPHGYFAQDDVLGYPDTG GQVVYILDQVRALESEMLSRIKKQGLDIIPRILIITRLLPDAVGTTCGQRLEKVYGTEHC HILRVPFRDEKRIVRKWISRFEVWPYLETYTEDVAHELAKELQSKPDLIVGNYSDGNIVA SLLAHKLGVTQCTIAHALEKTKYPESDIYWKKFEEKYHFSCQFTADLFAMNHTDFIITST FQEIAGSKDKVGQYESHTAFTLPGLYRVVHGIDVFDPKFNIVSPGADQTIYFPYTETSRR LTSFYPEIEELLYSSVENEEHICVLKDRNKPIIFTMARLDRVKNITGLVEWYGKNAKLRE LVNLVVVAGDRRKESKDLEEIAEMKKMYGLIETYKLNGQFRWISSQMNRVRNGELYRV ICDTKGAFVQPAVYEAFGLTVVEAMATGLPTFATLNGGPAEIIVHGKSGFHIDPYHGERA ADLLVEFFEKVKADPSHWDKISQGGLQRIEEKYTWTIYSQRLLTLTGVYGFWKHVSNL DRLESRRYLEMFYALKYRKLAESVPLAVE

>AAR19769.1 SUS [Beta vulgaris]

MASRLTRVPSLKERLDETLTAQRNEIISFLSKIASHGKGILQPHEVLSEFEAVADKHKLAD GPFGEVLRHTQETIVLPPWITLAVRPRPGIWEYIRVNVDALAVEELTPSQFLHVKEELVD GSTNGNFVLELDFEPFTASFPRPTLSKSIGNGVEFLNRHLSAKMFHDKESMRPLLDFLR MHHYKGKTIMLNDRIQNLDSLQAVLRKAEEFLTTIPADTPYSEFDHKFQEIGLERGWGD NAERVMDMIQLLLDLLEAPDSCTLEKFLGRIPMVFNVVILTPHGYFAQANVLGYPDTG 
GQVVYILDQVRALEHEMLLRIKQQGLDIIPRILIVSRLLPDAVGTTCGQRLEKVFGTEHS HILRVPFRTEKGIVRRWISRFEVWPYLETYTEDVANEIAGELQAKPDLIIGNYSDGNIVAS LLAHKLGVTQCTIAHALEKTKYPNSDIYWKSFEEKYHFSCQFTADLIAMNHTDFIITSTF QEIAGNKDTVGQYESHMAFTLPGLYRVVHGIDVFDPKFNIVSPGADLSIYFNYTEEKKR LTALHPEIEELLFSETQNEEHICVLKDRKKPIIFSMARLDRVKNMTGLVEWYGKNKKLR ELVNLVVVAGDRRKESKDTEEKEEMKKMYSLIEEYNLNGQFRWISAQMNRVRNGELY RYIADTRGAFVQPAYYEAFGLTVVEAMTCGLPTFATCHGGPAEIIVHGKSGFHIDPYHGD KAADLLVDFFEKSTADPSYWENISKGGLQRIEEKYTWKIYSDRLLTLAGVYGFWKYVS NLDRREARRYLEMFYALKYKKLAESVPLAIEDAN

>NP_001292638.1 SUS [Cucumis sativus]

MAERVLNRIHSLRERLDETLIAQRNEILGLLSKIEAKGKGILQHHELIAEFETIPEENRRK LADGAFGEVLRSTQESIVLPPWVALAVRPRPGVWEYIKVNVHALVVEELQASEYLRFK EELVDGSSNGNFVLELDFEPFNASFPRPTLSKSIGNGVEFLNRHLSAKLFHGKESMQPLL DFLRVHCYKGKTMMLNDRIQTLDAFQRVLRKAEEYLVTLAPETPYSEFANKFQEIGLER GWGDTAERVLEMIQLLLDLLEAPDPCTFEKFLGRIPMVFNVVILSPHGYFAQDNVLGYP DTGGQVVYILDQVRALEHEMLQRIKQQGLDITPRILIITRLLPDAVGTTCTQRLEKVFGT EHSHILRVPFRNEKGIVRKWISRFEVWPYLETYTEDVAQELTKELQGKPDLIIGNYSDGN IVASLLAHKLGVTQCTIAHALEKTKYPDSDIYWKTFDDKYHFSSQFTADLIAMNHTDFII TSTFQEIAGSKDTVGQYESHTAFTLPGLYRVVHGINVFDPKFNIVSPGADMSIYFPYTET EKRLTAFHPEIEELLYSEVENEEHLCVLKDRSKPIIFTMARLDRVKNITGLVEWYGKNDR LRELVNLVVVAGDRRKESKDNEEKAEMEKMYNLIKTYNLNGQFRWISAQMNRVRNGE LYRYIADTKGAFVQPAVYEAFGLTVVEAMTCGLPTFATCNGGPAEIIVDGKSGFHIDPYR GDRAAEILVDFFEKSKEDPTHWDEISQAGLKRIYEKYTWQIYSERLLTLTGVYGFWKHV SNLDRLESRRYLEMFYALKYRKLADSVPPAVDE

$>$ NP_001237525.1 SUS [Glycine max]

MATDRLTRVHSLRERLDETLTANRNEILALLSRIEAKGKGILQHHQVIAEFEEIPEENRQK LTDGAFGEVLRSTQEAIVLPPWVALAVRPRPGVWEYLRVNVHALVVEELQPAEYLHFK EELVDGSSNGNFVLELDFEPFNAAFPRPTLNKSIGNGVQFLNRHLSAKLFHDKESLHPLL EFLRLHSVKGKTLMLNDRIQNPDALQHVLRKAEEYLGTVPPETPYSEFEHKFQEIGLER GWGDNAERVLESIQLLLDLLEAPDPCTLETFLGRIPMVFNVVILSPHGYFAQDNVLGYP DTGGQVVYILDQVRALENEMLHRIKQQGLDIVPRILIITRLLPDAVGTTCGQRLEKVFGT EHSHILRVPFRTEKGIVRKWISRFEVWPYLETYTEDVAHELAKELQGKPDLIVGNYSDG NIVASLLAHKLGVTQCTIAHALEKTKYPESDIYWKKLEERYHFSCQFTADLFAMNHTDF IITSTFQEIAGSKDTVGQYESHTAFTLPGLYRVVHGIDVFDPKFNIVSPGADQTIYFPHTET SRRLTSFHPEIEELLYSSVENEEHICVLKDRSKPIIFTMARLDRVKNITGLVEWYGKNAKL RELVNLVVVAGDRRKESKDLEEKAEMKKMYGLIETYKLNGQFRWISSQMNRVRNGEL YRVICDTRGAFVQPAVYEAFGLTVVEAMTCGLPTFATCNGGPAEIIVHGKSGFHIDPYHG DRAADLLVDFFEKCKLDPTHWDKISKAGLQRIEEKYTWQIYSQRLLTLTGVYGFWKHV SNLDRRESRRYLEMFYALKYRKLAESVPLAAE

>NP_001275286.1 SUS [Solanum tuberosum]

MAERVLTRVHSLRERVDATLAAHRNEILLFLSRIESHGKGILKPHELLAEFDAIRQDDKN 
KLNEHAFEELLKSTQEAIVLPPWVALAIRLRPGVWEYIRVNVNALVVEELSVPEYLQFK EELVDGASNGNFVLELDFEPFTASFPKPTLTKSIGNGVEFLNRHLSAKMFHDKESMTPLL EFLRAHHYKGKTMMLNDRIQNSNTLQNVLRKAEEYLIMLSPDTPYFEFEHKFQEIGLE KGWGDTAERVLEMVCMLLDLLEAPDSCTLEKFLGRIPMVFNVVILSPHGYFAQENVLG YPDTGGQVVYILDQVPALEREMLKRIKEQGLDIIPRILIVTRLLPDAVGTTCGQRIEKVY GAEHSHILRVPFRTEKGIVRKWISRFEVWPYMETFIEDVAKEISAELQAKPDLIIGNYSEG NLAASLLAHKLGVTQCTIAHALEKTKYPDSDIYWKKFDEKYHFSSQFTADLIAMNHTD FIITSTFQEIAGSKDTVGQYESHMAFTMPGLYRVVHGINVFDPKFNIVSPGADINLYFSYS ETEKRLTAFHPEIDELLYSDVENDEHLCVLKDRTKPILFTMARLDRVKNLTGLVEWYAK NPRLRGLVNLVVVGGDRRKESKDLEEQAEMKKMYELIETHNLNGQFRWISSQMNRVR NGELYRYIADTKGAFVQPAFYEAFGLTVVEAMTCGLPTFATNHGGPAEIIVHGKSGFHID PYHGEQAADLLADFFEKCKKEPSHWETISTGGLKRIQEKYTWQIYSERLLTLAAVYGF WKHVSKLDRLEIRRYLEMFYALKYRKMAEAVPLAAE

$>$ CAA46017.1 SUS [Oryza sativa]

MAAKLARLHSLRERLGATFSSHPNELIALFSRYVNQGKGMLQRHQLLAEFDALIEADK EKYAPFEDILRAAQEAIVLPPWVALAIRPRPGVWDYIRVNVSELAVEELSVSEYLAFKEQ LVDGHTNSNFVLELDFEPFNASFPRPSMSKSIGNGVQFLNRHLSSKLFQDKESLYPLLNF LKAHNHKGTTMMLNDRIQSLRGLQSSLRKAEEYLMGIPQDTPYSEFNHRFQELGLEKG WGDCAKRVLDTIHLLLDLLEAPDPANLEKFLGTIPMMFNVVILSPHGYFAQSNVLGYPD TGGQVVYILDQVRALENEMLLRIKQQGLDITPKILIVTRLLPDAVGTTCGQRVEKVIGTE HTDILRVPFRSENGILRKWISRFDVWPFLETYTEDVANEIMREMQAKPDLIIGNYSDGNL VATLLAHKLGVTQCTIAHALEKTKYPNSDIYLDKFDSQYHFSCQFTADLIAMNHTDFIIT STFQEIAGSKDTVGQYESHIAFTLPGLYRVVHGIDVFDPKFNIVSPGADMSVYFPYTEAD KRLTAFHPEIEELLYSEVENDEHKFVLKDKNKPIIFSMARLDRVKNMTGLVEMYGKNAH LRDLANLVIVCGDHGNQSKDREEQAEFKKMYGLIDQYKLKGHIRWISAQMNRVRNGE LYRYICDTKGVFVQPAFYEAFGLTVIEAMTCGLPTIATCHGGPAEIIVDGVSGLHIDPYHS DKAADILVNFFEKCKQDSTYWDNISQGGLQRIYEKYTWKLYSERLMTLTGVYGFWKY VSNLERRETRRYIEMFYALKYRSLASAVPLAVDGESTSK

$>$ CAD85125.1 SUS [Nitrosomonas europaea ATCC 19718]

MTTIDTLATCTQQNRDAVYTLLRRYFTANRTLLLQSDLREGLLQTEQDCGQSDMLRAF VFRLQEGIFSSPWAYLALRPEIAKWEFMRIHQEHLIPEKLTISEFLKFKETVVKGEATESV LEVDFGPFNRGFPRLKESRSIGQGVIFLNRKLSSEMFSRIEAGHTSLLHFLGVHAIEGQQL MFSNNSHDIHAVRNQLRQALEMLETLDGTTPWIELAPKMNQLGFAPGWGHNANRVAE TMNMLMDILEAPSPSALEEFLACIPMISRLLILSPHGYFGQDNVLGLPDTGGQVVYILDQ VRALEKEMHDRLQLQGVQVEPKILIVTRLIPDAGDTTCNQRLEKVSGCTNTWILRVPFR KHNGEIIPHWISRFEIWPHLEIFAGDVEREALAELGGHPDLIIGNYSDGNLVATLLSRRLG VTQCNIAHALEKTKYLHSDIYWQENEDKYHFSCQYTADLLAMNSADFIVTSTYQEIAG TREAEGQYESYQAFSMPDLYRVIHGIDLFDPKFNIVSPGANADIYFPYSDPNRRLHSLIPE IESLIFDDATNLPARGYLQDPDKPLIFTMARLDRIKNITGLVELYAASPRLRSLANLVIVG GKIDPQHSSDHEEQEQIHRMHQLMDEHELDQQVRWLGMRLDKNLAGELYRYIADKRG IFVQPALFEAFGLTIIEAMASGLPTFATRYGGPLEIIQNNRSGFHIDPNQGAATADLIADFF EKNLENPQEWERISQGALDRVASRYTWKLYAERMMTLSRIYGFWKFVSGLEREETDRY 
LNMFYHLQFRPLANRLAHEI

>A0A059ZV61.1 SUS [Acidithiobacillus caldus ATCC 51756]

MIEALRQQLLDDPRSWYAFLRHLVASQRDSWLYTDLQRACADFREQLPEGYAEGIGPLE DFVAHTQEVIFRDPWMVFAWRPRPGRWIYVRIHREQLALEELSTDAYLQAKEGIVGLG AEGEAVLTVDFRDFRPVSRRLRDESTIGDGLTHLNRRLAGRIFSDLAAGRSQILEFLSLH RLDGQNLMLSNGNTDFDSLRQTVQYLGTLPRETPWAEIREDMRRRGFAPGWGNTAGR VRETMRLLMDLLDSPSPAALESFLDRIPMISRILIVSIHGWFAQDKVLGRPDTGGQVVYI LDQARALEREMRNRLRQQGVDVEPRILIATRLIPESDGTTCDQRLEPVVGAENVQILRV PFRYPDGRIHPHWISRFKIWPWLERYAQDLEREVLAELGSRPDLIIGNYSDGNLVATLLSE RLGVTQCNIAHALEKSKYLYSDLHWRDHEQDHHFACQFTADLIAMNAADIIVTSTYQEI AGNDREIGQYEGHQDYTLPGLYRVENGIDVFDSKFNIVSPGADPRFYFSYARTEERPSFL EPEIESLLFGREPGADRRGVLEDRQKPLLLSMARMDRIKNLSGLAELYGRSSRLRGLAN LVIIGGHVDVGNSRDAEEREEIRRMHEIMDHYQLDGQLRWVGALLDKTVAGELYRVVA DGRGVFVQPALFEAFGLTVIEAMSSGLPVFATRFGGPLEIIEDGVSGFHIDPNDHEATAER LADFLEAARERPKYWLEISDAALARVAERYTWERYAERLMTIARIFGFWRFVLDRESQV MERYLQMFRHLQWRPLAHAVPME

$>$ I7A3T6.1 SUS [Melioribacter roseus P3M-2]

MIKDIYKTAETFHNDFYDFLKAVSTQPKKLMITGELINLYVASGYDKNSGLYEFIEKIQET ISLDHSVILDVRIKIASIKFYRISLEEFLIEEISSKEFLIYKETVAKPDTLNTTLNLNFKPFYD KSPAVRDIKYIGSGVEYLNRFLSSQMFTNEERWKKNLFDFIRLHNFNGEQLILNDRIKDT KHLNNQINAALAKLGNHPANTPYENIKHILQELGFEKGLGKDAGTITHNLNLLDQLLNS PDHNALAEFISSIPMILNIAIISPHGFFGQEGVLGLPDTGGQVVYILDQVKALEKQLIDSL KKSGLNLLPKIIVLTRLIPNARGTTCNQRLEKIYGAKNSWILRVPFREYNKRVTDEWISR FEIWPYLEDFAEDSYTALLAEFKKRPDLIIGNYSDGNLVAYLLAKKFKVTQCGIAHALEK SKYLYSALYWYDLEKYYHFSMQFTADLLAINSADFLITSSFQEIAGTEKSIGQYESYMHF TMPGLYRVENGVNPFHVKFNIVSPGVNEKIYFPYPKTKWRLKETKRRIENLFFSNSEDP DVIGWLDNPEKTPIFTMSRLDRIKNISFLVRCFGESEELQQTSNLIVVAGKIDETMTDDY EEKEQIRLMHELITKYKLHNKIRWIGKLLPKDESGEAYRIIAERRGIFVQPALFEGFGLTV LEAMTSGLPVFATKYGGPLEIIQNGVNGFHIDPVNQEETTEKIVRFLSDSYIDSSVWDKL SKAAIKRVTEKYSWKLYSKRLLSLAKLYGFWKYATNLEHEDINAYLDLIYHTIYKSRAKI LLEEHMKR

>AFY42156.1 SUS [Nostoc sp.PCC 7107]

MNELLQAVLNSDEKSVLQELIFSLTNSGKQYFLRNEILQVFADYCHNSQKPAYFYYSSSI GKLIHYTHEIILSEGNVWFVIRPKIASQEVWRLNGDLSQCELMSPQAYLDESDRLVNHY QPHILEIDLAPFYEDSPSVDDPRNIGQGLAFLNHYLCDQLVNDPQHWLEVLFQALRRVQ YDGRRLLIGDRISSGLQFAQQIKQAIKFLSDRPAKEPYEKFHFHLQDLGLEPGWGNTAA RVSETLSLLDRLIDTPQPAILEAFVARVPVVFRVVLISIHGWVGQQDVMGRDETLGQVIY VLEQARSLENKLHEEIALAGLDILGIQPHVIILTRLIPNCEGTSCGLKLEKVEDTENAWIL RVPFAEFNPEITNNWISKFEIWPYLESFTNDAEKELIAVFKGRPNLIIGNYSDGNLVASLLS HRLKVTQCNIAHSLEKPKYLFSNLYWHNLEDQYHFSAQFTADLISMNAADFIITSSYQEI VGTPDGMGQYESYKCFTMPELYHVVDGIDLFSPKFNLVPPGVNQKIFFPYTQKENRNF 
HQSKQVEDLIFNRQDLQIVGVLDEPNKQPIFAVATLSSIKNLTGLVECFAQSEELQKRCNL IILTSKLHPEEAANLEEAAEIQRLHDIINQHHLHNHLRWVGMRLTGVDIGEAYRVIADRQ GIYVHFARFESFGRSILEAMISGLPTFATQFGGALEIIENQEDGFIINPTDLGGTAQKIISFL DECENHPQHWQEVSEWMSQRIINKYNWSSHTSQLLLMAKMFSFWNFVSPENNEARDR YMESLFHLIFKPRAEKILEQHIH

>WP_131120612.1 SUS [Westiellopsis prolifica]

MYELIQTVLSSDEKTILRQLISDLSASGKRYFLRNEILHAFAEYCHQFQKPAYFFHSSSLG TLIHYTHEIILEGDDIWLLLRPRIGSQEVWRLLSADTSKFELQTPQALLDVRDRCVNRYQ SQILEINFYPFDRNIPSIDDPRNIGQGLAFLNRYLCSQVLSDRNYWLDVLFDALHRLEYD HKQLLIGDRIKTGLQLYKQIKQAIKIISQRPPEEPYEKFSEQLQTLGLEPGWGNTASRVRE TLELFDRLLETPQPAILEAFVDRVPAVFRVVLVSIHGWITQDDAVGRPETLGQVVYVLEQ ARSLENKLREEIKLAGLESLGIQPQLVILTRLIPNCEGTQCNLRLEKIEGTENAWILRVPFR EFNPQVTQNWISKYEIWPYLETFAIDAEKQLLAQLGGSPDLIIGNYSDGNLVAFLLARSL KVTQCNIAHSLEKPKHLFSNLYWQDLEEQYHFSAQFTADLISMNAADFILTSSYQEIVGT PDTLGQYESYKMFSMPELYHVVDGINLLSSRFNFVPPGVDENIFFPYNQKDKRDHNLSL KINDLLFNRSDDHIFGHLNHPNKRPICAIAHITPVKNLSGLVECFGQSQELRKQCNLILVT NKLHLSEATNSKEATELEKIHNLINQYNLYSQIRWVGMRLPKPELGEIYRAMADRQGIF VHFASFEAFGRPILEAMSSGLPTFATQFGGAAELIDDGECNFHINPTDIEGTANKILQFLD QCNTNPEHWYEISERVIQRVHNKYNWQMHTKQVLLLAKIYRFWDFINKENREALLRY VDTLYHLVFKPRSEKILEEHMQR

$>$ AFZ01160.1 SUS [Calothrix sp. PCC 6303]

MHELAQTVLNSDEKVALRQLISTLGTSGKRYLLRNEILQAFGDYCQQTEKPAYFFHVSG LGKLINYTHEIILEEESTWFLVRPWIASQEVWRLGADMESFERMTPQALLNVRDRIVNR FQPQILEIDLEPFYQGSPRIDDARNVGQGLAFLNHYLCSQLETDPEHWLEVLFQVLHRL EYDGLPLLISDRITSGKELVEQVKAALTILNQRSPQETYDKFRFDLQALGLEPGWGNTAS RVRETLELFNRLIDNPEPGVVEAFVARIPAIFRVTLISIHGWVGQEGVAGKPETAGQVMY VIEQARNLEKKLREEIKLAGLDLLGIKPHVIILTRLIPNCEGTACDMRLEKLQGSDNAWIL RVPFAEHNLQVTQNWISKYEIWPYLDHFALEAEKQLLAEFRGRPNLIIGNYSDGNLVAS LLARRLKATQCNIAHALEKPKNLFSNLYWQDLEERYHFSAQFTADLISMNAADFIIASSY QEIVGTPDSIGQYESYKCFTMPSLYHVVDGIDLFNPKFNVVPPGVNENIFFAPSQTGNRD DNCRSQICQTLFTREDPQILGNLKDPKKRPIFAIAPIDAIKNLTRLTECFSKSPELQEHCNL ILLTSKLHPDQTTHPEQAEEIAKLHELINHYHLEGKIRWLGLRLSNLDLGETYRAIADYQ GIFVHFAHFEPFGRTILEAMISGLPTFATQFGGVSEIIEDKDVFLINPTDLEGTTQKIVQFLE TCDRIPEYWQETSQRVIERIQNKFNWDSHCKQLLLIAKIYKFWDFAIPENREARMRYLE TLYHLVFKPRAAEILEKHSHIKSE

>AFZ34482.1 SUS [Stanieria cyanosphaera PCC 7437]

MSYLIEAVLNSEEKNNLREFISQLQESDQRYLLRNEILSSFDSFSTTKSEAQPYFSSNLGK LIYYTQEIILEKESICLIIRPKIAQQEAYRIFEDLNYEAMTVQQLLDTRDRFVNHFHPDEG DVFEIDFRPFYDYSPTIRDPKNIGRGVQFLNRYLSSKIFQDPNQWLEALYSFLSLHSYNG NTLLINGRIKDRKQLSDRIKQALNLVSGFPKSKPYEEFRFELQNFGFEPGWGNTAGRVR ETLEILDELIDSPDYKVLEDFLSRIPMIFRIVLVSVHGWFAQEGVLGRPDTGGQVVYVLD 
QARSLEKQLQEDISLAGLDSLNIEPKVIILTRLIPNSDGTRCNERLEKVHGTDNGWILRVP LREFNPNMTQNWISRFEIWPYLETYAVDAEKELYQEFRGIPDLIIGNYSDGNLVSFLLAR RLKVTQFTIAHALEKSKYLFSNLYWQDLEPNYHFSLQFTADLIAMNAANCIISSTYQEIA GRSDSVGQYESYDNFTMPDLYHVVNGIELFSPKFNIVPPGVNENVYFPYTRSEDRPPSKI EKLEELLFSTEDPSFIFGKLEDPGKRPLFSMARLDRIKNLTGLAECFANSPELQERCNLIL VAGKIHGAETQDNEEREEIEKMYRIIEQHNLHGKMRWLGLRLPKSDSGEIYRVIADRRG VFVQPALFEAFGLTILEAMISGLPTFGTQFGGPLEIIQDGINGFYINPTNLEETASKILEFVS KCDQNPDYWHEISNQGIERVYSTYTWKIHTTKLLSLARIYGFWNYTSKEKREDLLRYIE ALFYLIYKPRAKELLEQHWQR

$>$ EEG77295.1 SUS [Dethiobacter alkaliphilus AHT 1]

MENLFSQHREAVYLLLRHYFKLDRTFLLGSDLRDELENFLSNQDEETVKKLEPLSKLIK DAQEAILSDPWVYLATRPNVARWKYYRFHMHDMLFNEIHVSEFLAFKERQVNGHDDE EWMLELDFDPFNRDFPKLKEARSIGNGLQFLNRHLSSRFFHEQAKAQEILLEFLRRHHIR DRNLMLNGRIKTIKALRSALRSADEHLENQSEDTTWHDVGPALQELGFEPGWGRDLPR IRETMRLLSDILEAAEPGNLEMFLGRVPMIFNIVILSPHGYFGQDNVLGLPDTGGQVVYI LDQVRALEEEMCSRLYDQGLDLMPQILVVTRLIPEAGNTTCDQRLEDIVGTENARILRV PFRNPDGQVVRPWISRFNIWPYLERFSQDAEKEVLAELGAKPDLILGNYSDGNLVATLM AKKIGATQCNIAHALEKPKYLYSDLYWKDNEEQYHFSCQFTADLIAMNAADFIITSTFQ EIAGKKDTVGQYESYNAFTMPGLYRVVNGINIFDPKFNIVSPGADPVSYFPYTEKKRRL YALHDEIEEMVYSGERSDIRGHFTDKEKPLLYTMARLDTIKNITGLVEWYGKNERLRKS ANLLIKAGHVDPALSQDTEEKAQIARMHQLMDEYELDGQVRWLGFHLEKNLSSEMYR FVADKRGAFIQPALFEAFGITVIEAMISGLPTFATCYGGPSEIIEEGVSGFHIDPNHGERSA NKIADFMEKSATDPSHWDSISQGGIERVLDRYTWELYARRLITLSCIYGFWKYVSDLRR VETKQYLDMFYGLQYRPLANSVELAD 
Table S6. ${ }^{1} \mathrm{H}$ NMR, ${ }^{13} \mathrm{C}$ NMR data of GA-glucosylated products

GA-30-O-glucose: ${ }^{1} \mathrm{H}-\mathrm{NMR}$ (Methanol- $\left.d 4,700 \mathrm{MHz}\right) \delta 5.63(1 \mathrm{H}, \mathrm{s}, \mathrm{H}-12), 5.53$ $(1 \mathrm{H}, \mathrm{d}, J=8.2 \mathrm{~Hz}, \mathrm{Glc}-1-\mathrm{H}), 3.88(1 \mathrm{H}, \mathrm{dd}, J=12.0,2.0 \mathrm{~Hz}, \mathrm{Glc}-6-\mathrm{H} 1), 3.72(1 \mathrm{H}$, $\mathrm{dd}, J=12.0,4.9 \mathrm{~Hz}$, Glc-6-H2), 3.46-3.43 (1H, m, Glc-3-H), 3.42-3.40 (1H, m, Glc5-H), 3.38-3.34 (1H, dd, Glc-4-H), 3.33 (1H, td, J = 3.5, $2.0 \mathrm{~Hz}, \mathrm{Glc}-2-\mathrm{H}), 3.19(1 \mathrm{H}$, $\mathrm{dd}, J=11.8,4.5 \mathrm{~Hz}, \mathrm{H}-3) ;{ }^{13} \mathrm{C}$ NMR (Methanol- $\left.d 4,176 \mathrm{MHz}\right) \delta 201.37(\mathrm{C}-11$ ), 175.54(C-30), 171.48(C-13), 127.57(C-12), 94.32(Glc-C-1), 78.00(C-3), 77.54(GlcC-5), 76.90(Glc-C-3), 72.69(Glc-C-2), 69.82(Glc-C-4), 61.75(C-9), 61.12(Glc-C-6), 54.79(C-5), 48.13(C-18), 45.34(C-8), 43.83(C-20), 43.18(C-14), 40.91(C-19), 38.91(C-1), 38.83(C-4), 37.21(C-22), 36.93(C-10), 32.41(C-7), 31.57(C-17), 30.51(C-21), 27.57(C-28), 27.27(C-23), 26.79(C-29), 26.42(C-2), 26.19(C-15), 26.06(C-16), 22.39(C-27), 17.88(C-26), 17.20(C-6), 15.54(C-25), 14.92(C-24).

GA-30-O-glucose-3-O-glucose: ${ }^{1} \mathrm{H}-\mathrm{NMR}$ (Methanol- $\left.d 4,700 \mathrm{MHz}\right) \delta 5.63(1 \mathrm{H}, \mathrm{s}$, H-12), 5.53 (1H, d, $J=8.2$ Hz, 30-O-Glc-1-H), 4.35 (1H, d, $J=7.8$ Hz, 3-O-Glc-1H), 2.47 (1H, s, H-9), 0.81 (1H, d, $J=11.4 \mathrm{~Hz}, \mathrm{H}-5) ;{ }^{13} \mathrm{C}$ NMR (176 MHz, MeOD) $\delta$ 201.34(C-11), 175.54(C-30), 171.42(C-13), 127.60(C-12), 105.32(3-O-Glc-C-1), 94.31(30-O-Glc-C-1), 89.06(C-3), 77.52(30-O-Glc-C-5), 76.89(30-O-Glc-C-3), 76.85(3-O-Glc-C-3), 76.27(3-O-Glc-C-5), 74.25(3-O-Glc-C-2), 72.68(30-O-Glc-C2), 70.23(3-O-Glc-C-4), 69.83(30-O-Glc-C-4), 61.78(C-9), 61.40(3-O-Glc-C-6), 61.14(30-O-Glc-C-6), 55.11(C-5), 45.38(C-18), 43.83(C-8), 43.18(C-20), 40.93(C14), 39.14(C-19), 38.92(C-4), 37.22(C-1), 36.68(C-22), 32.43(C-10), 31.58(C-7), 30.52(C-17), 27.61(C-21), 27.11(C-28), 26.82(C-23), 26.21(C-29), 26.07(C-15), 25.63(C-16), 22.46(C-2), 19.57(C-27), 17.93(C-26), 17.05(C-6), 15.65(C-25), 15.64(C-24). 


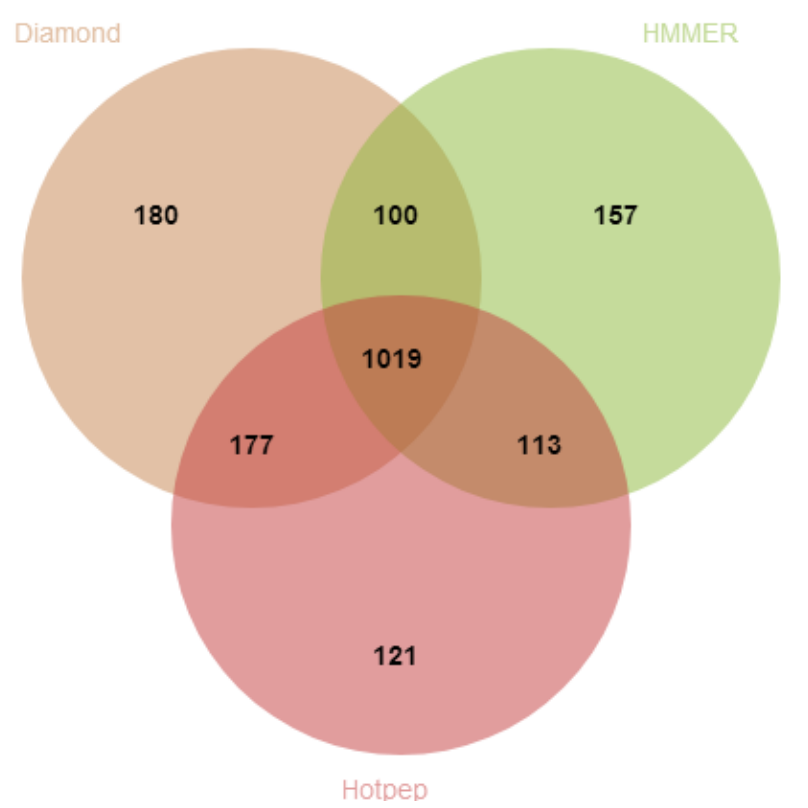

Figure S1. Venn chart of CAZymes in G. uralensis 


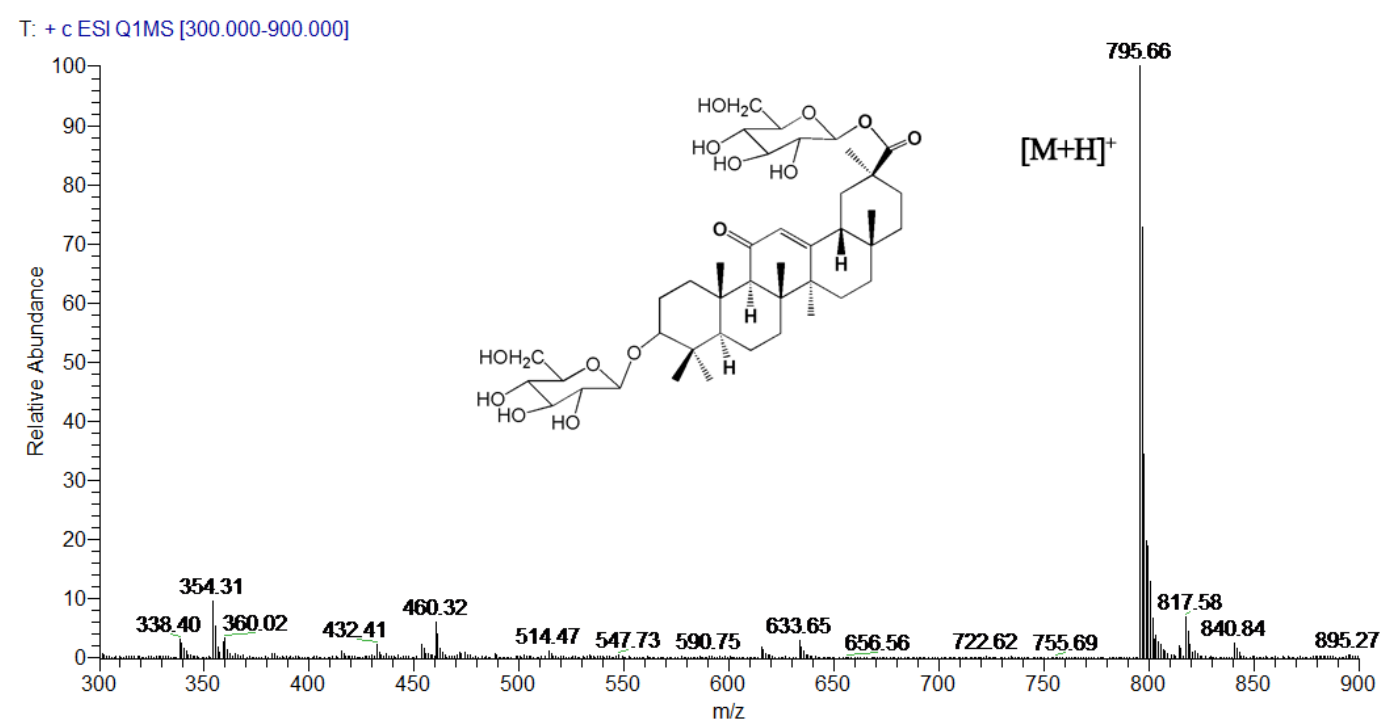

Figure S2. Positive ion MS spectra for GA-30-O-glucose-3-O-glucose 


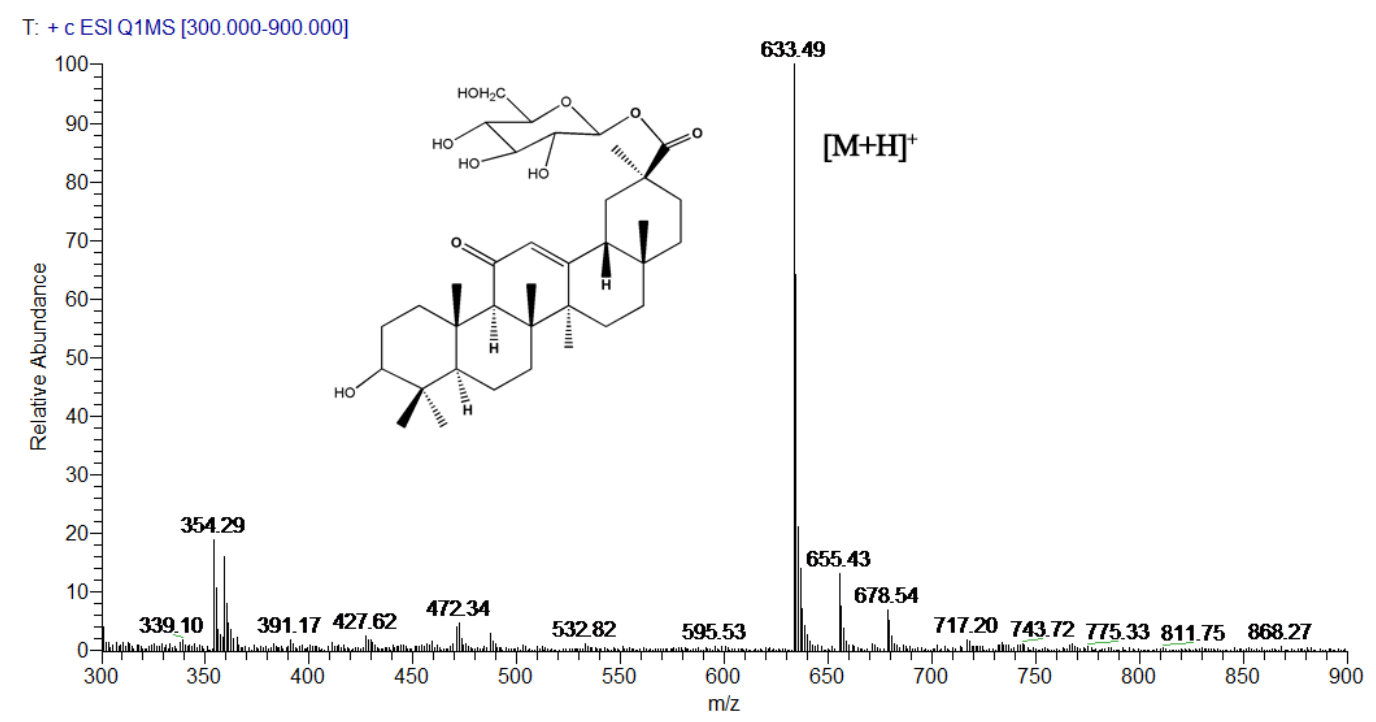

Figure S3. Positive ion MS spectra for GA-30-O-glucose 


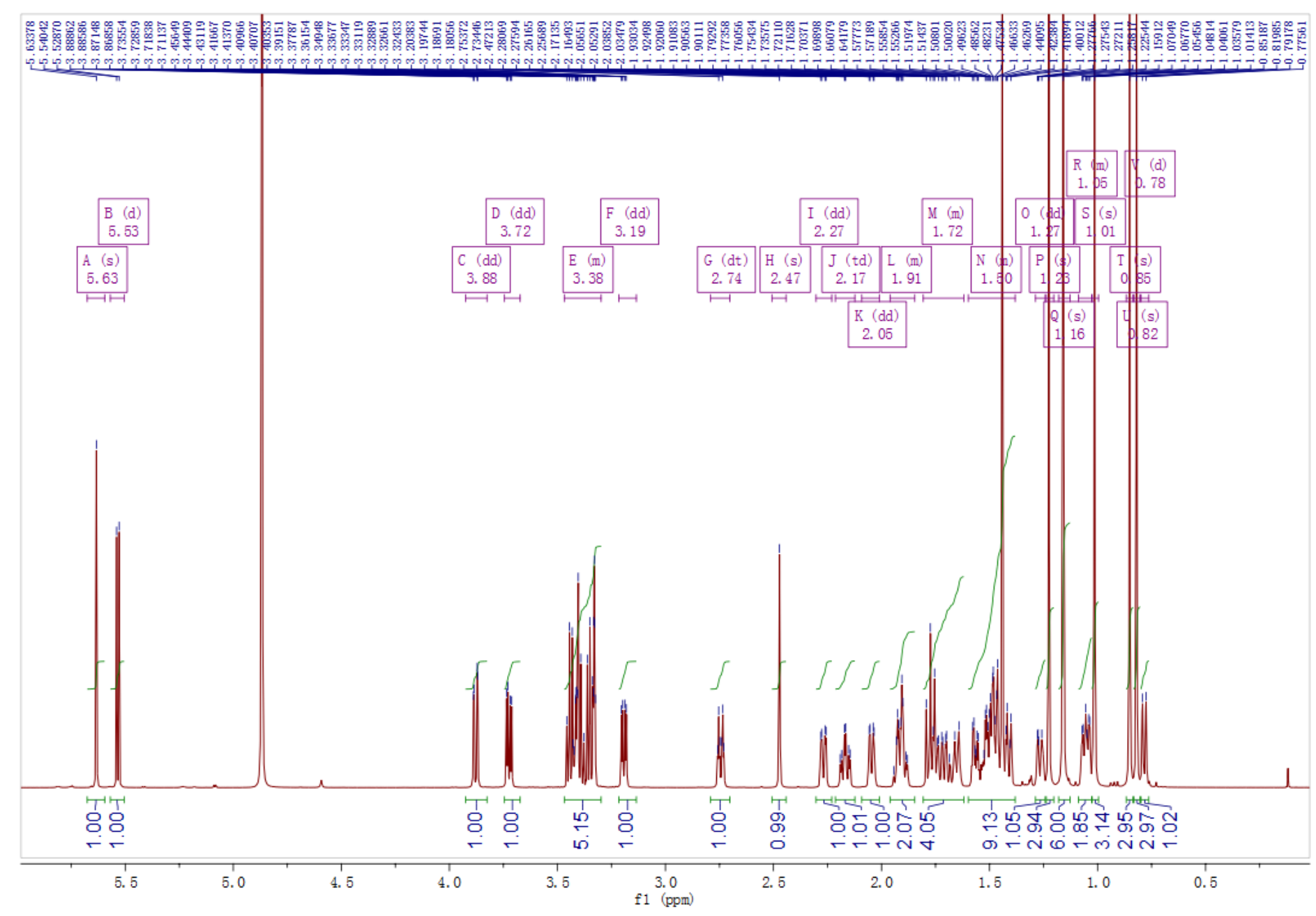

Figure S4. ${ }^{1} \mathrm{H}$ NMR spectrum of GA-30-O-glucose (Methanol-d4) 


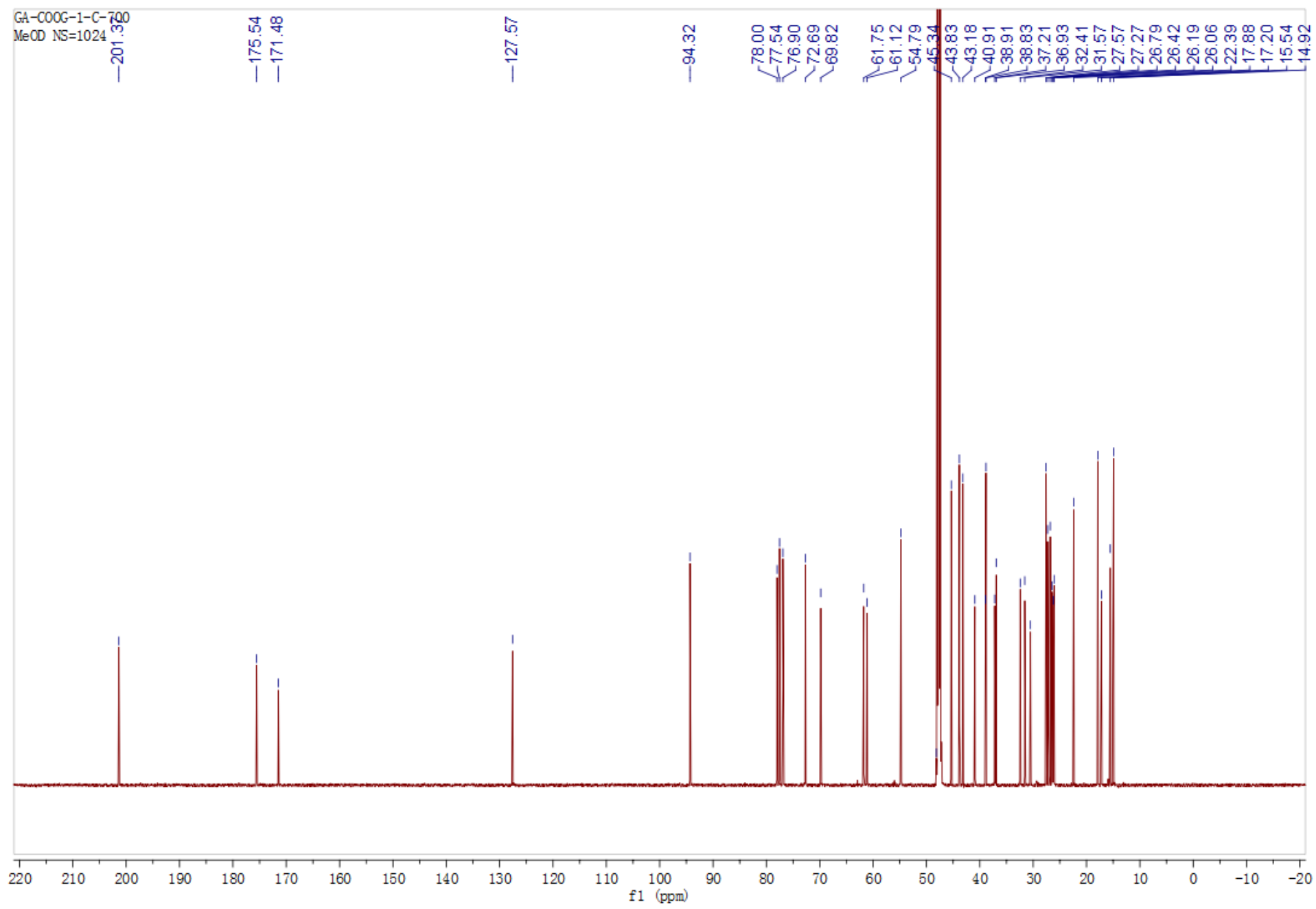

Figure S5. ${ }^{13} \mathrm{C}$ NMR spectrum of GA-30-O-glucose (Methanol- $d 4$ ) 


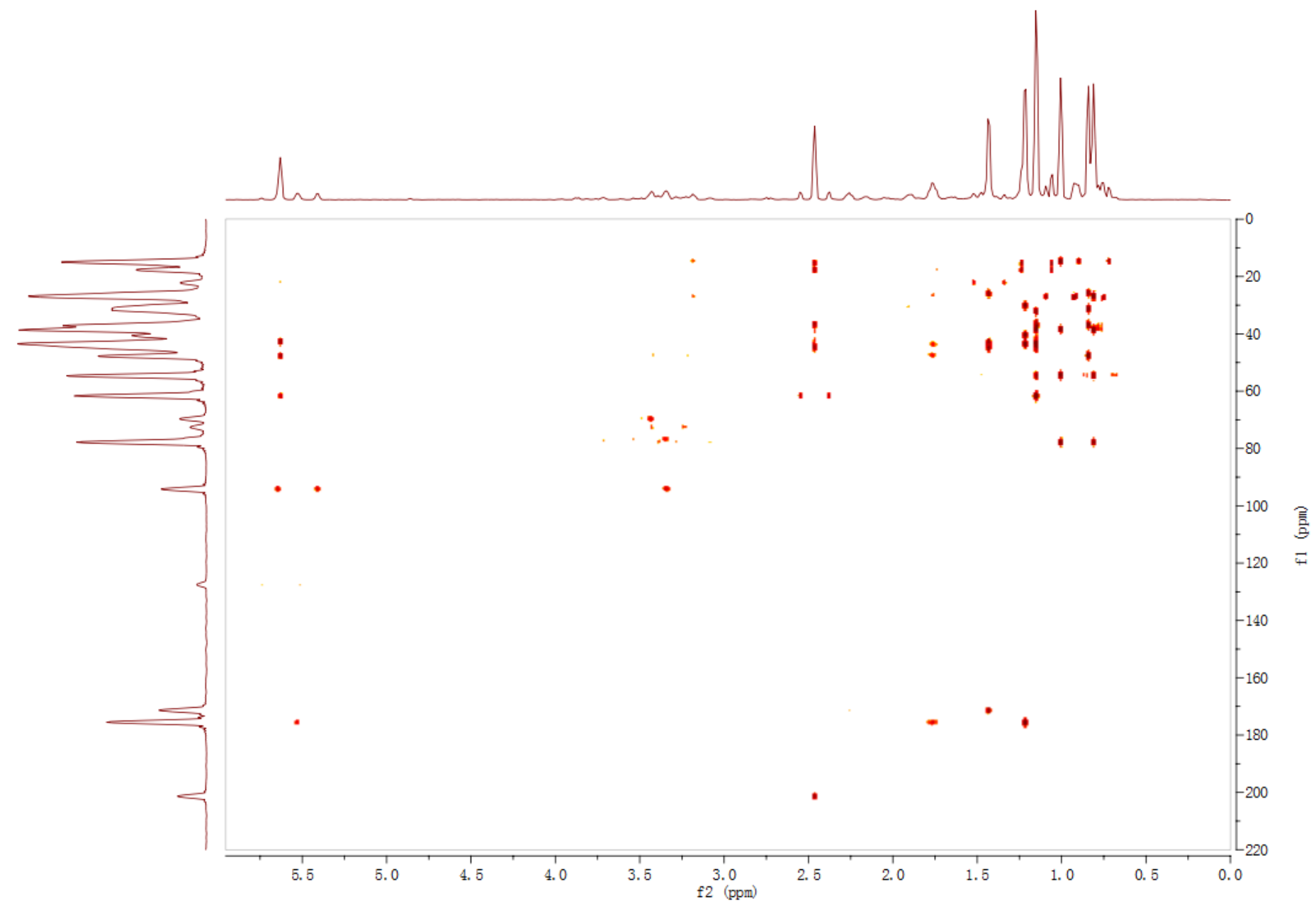

Figure S6. HMBC spectrum of GA-30-O-glucose (Methanol- $d 4$ ) 


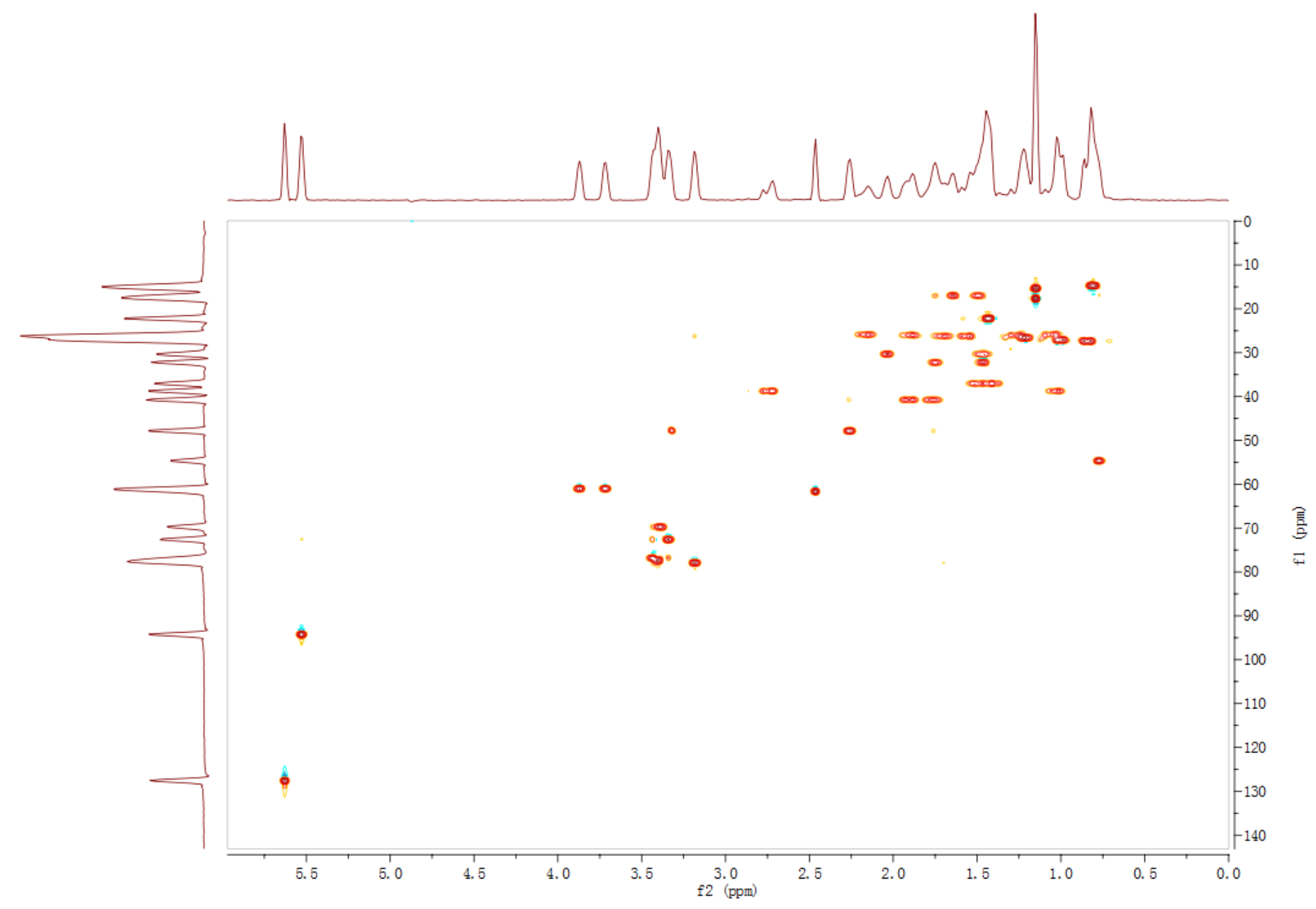

Figure S7. HSQC spectrum of GA-30-O-glucose (Methanol- $d 4$ ) 


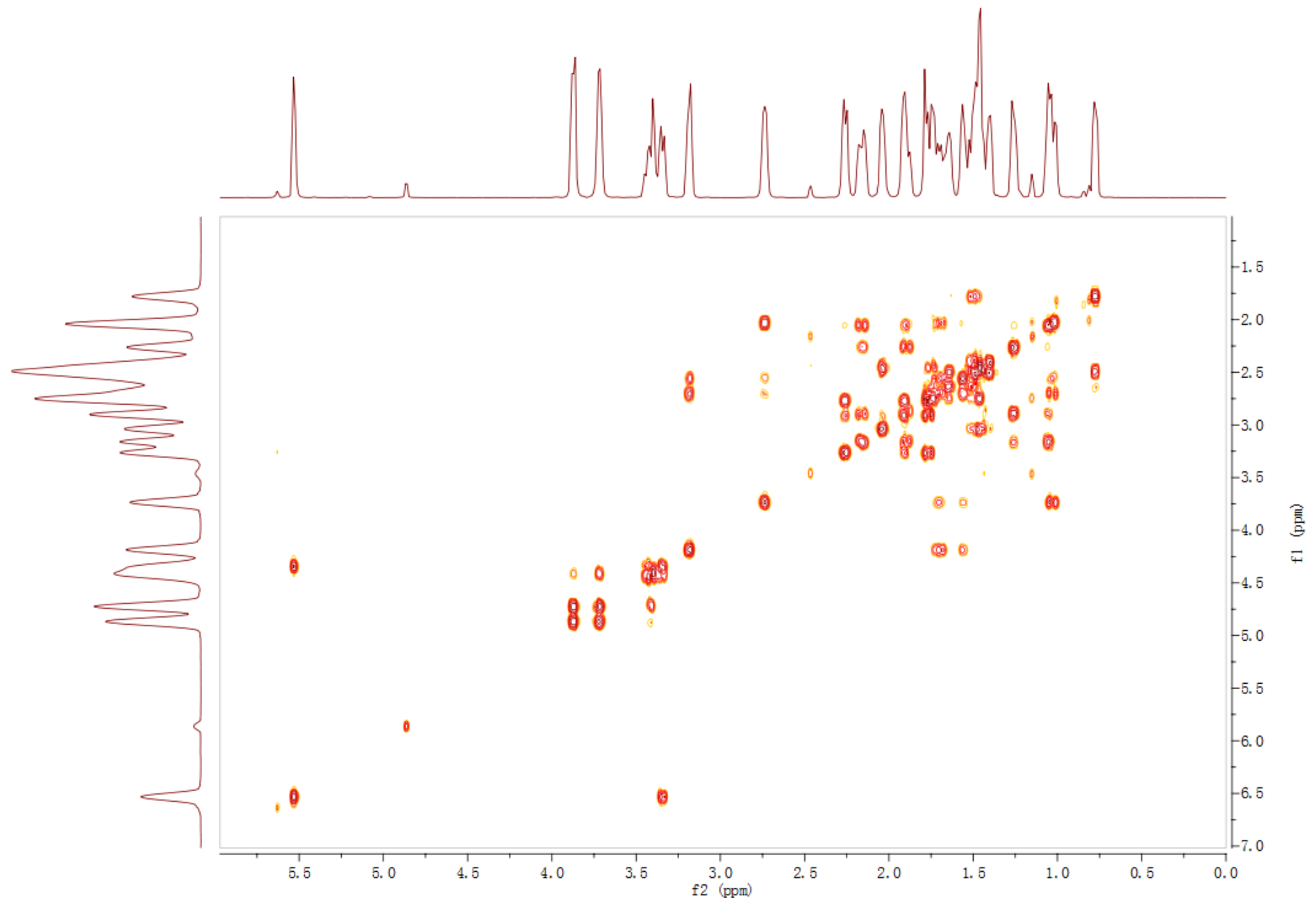

Figure S8. COSY spectrum of GA-30-O-glucose (Methanol- $d 4$ ) 


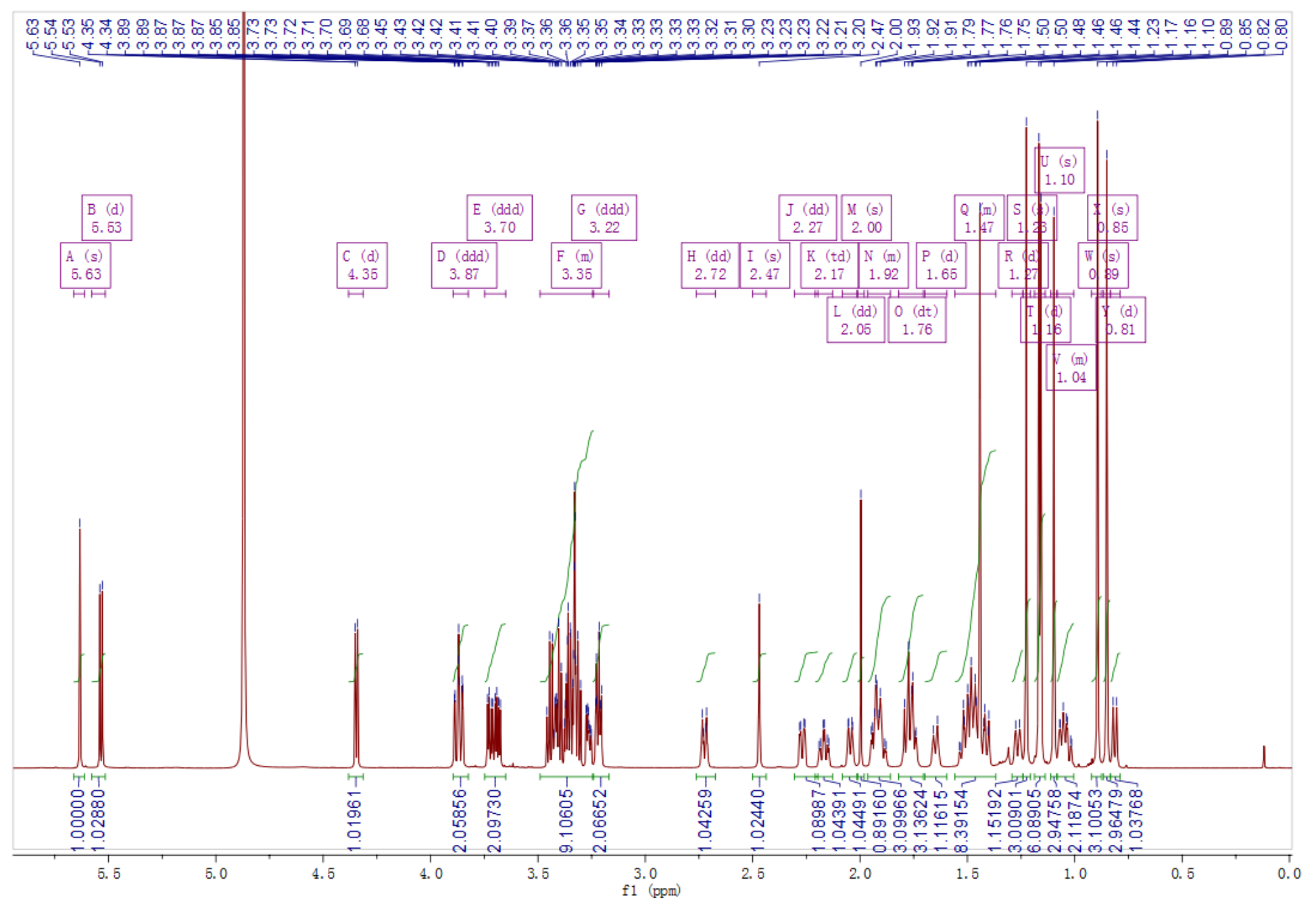

Figure S9. ${ }^{1} \mathrm{H}$ NMR spectrum of GA-30-O-glucose-3-O-glucose (Methanol- $d 4$ ) 


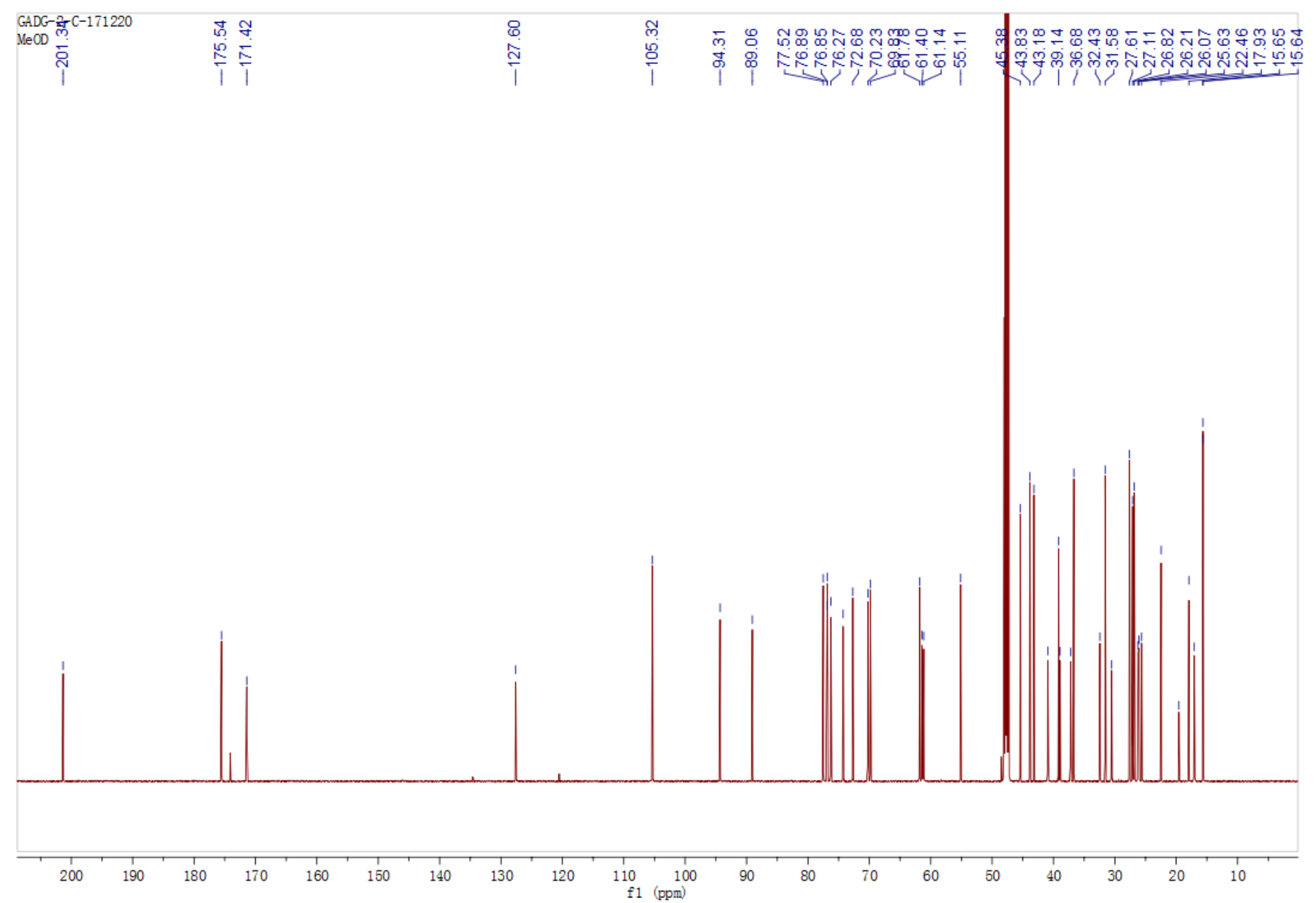

Figure S10. ${ }^{13} \mathrm{C}$ NMR spectrum of GA-30-O-glucose-3-O-glucose (Methanol- $d 4$ ) 


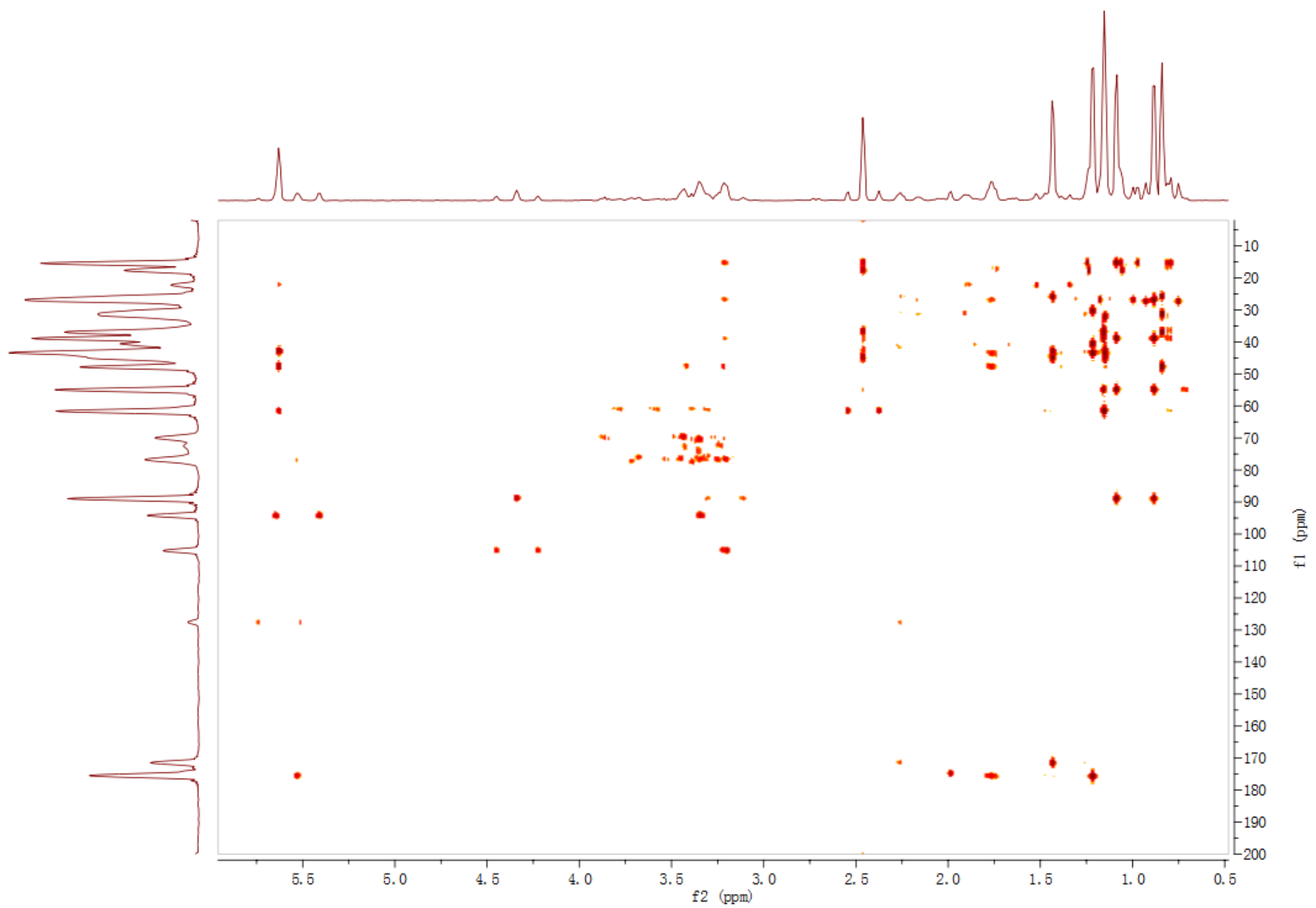

Figure S11. HMBC spectrum of GA-30-O-glucose-3-O-glucose (Methanol- $d 4$ ) 


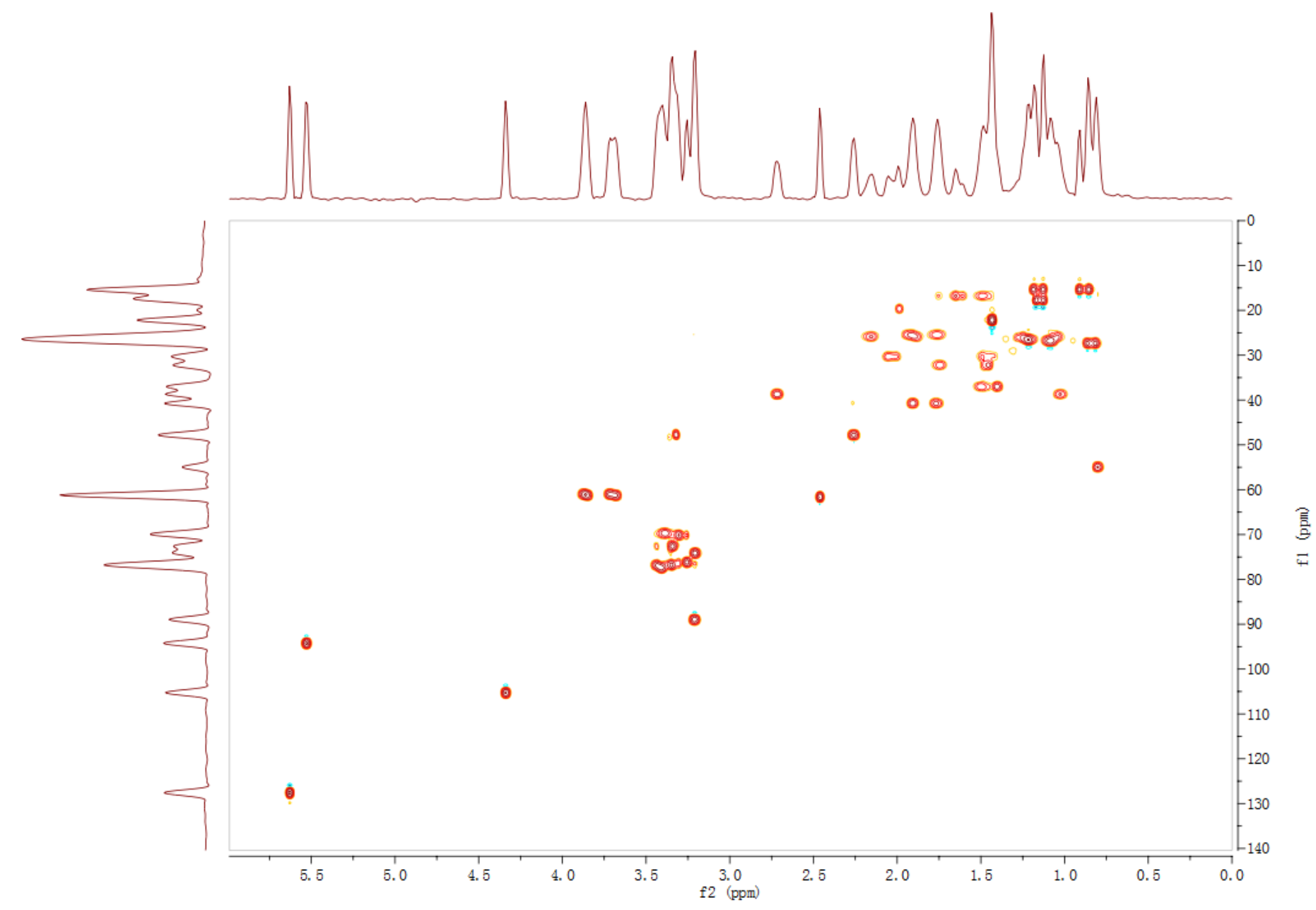

Figure S12. HSQC spectrum of GA-30-O-glucose-3-O-glucose (Methanol- $d 4$ ) 


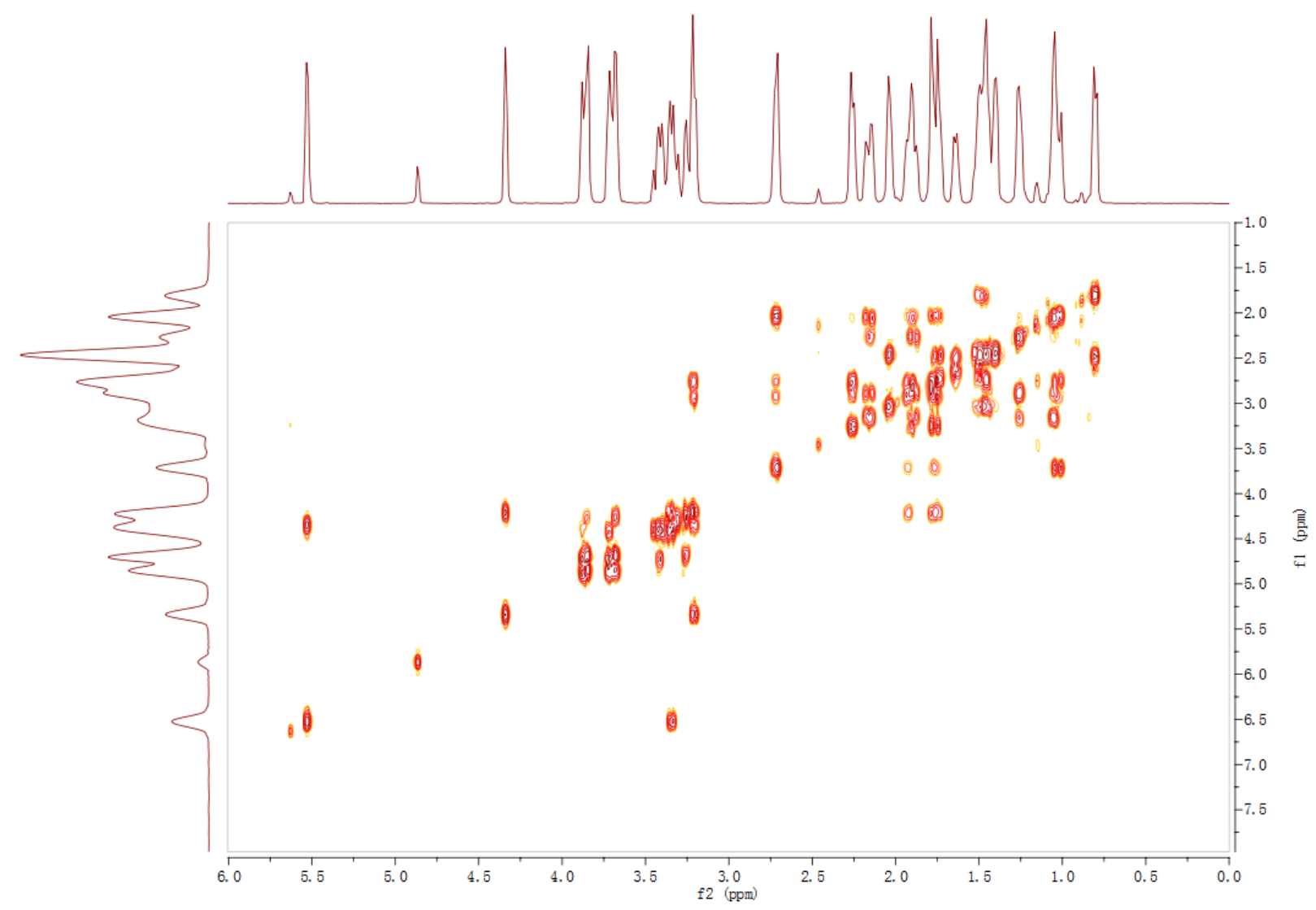

Figure S13. COSY spectrum of GA-30-O-glucose-3-O-glucose (Methanol- $d 4$ ) 


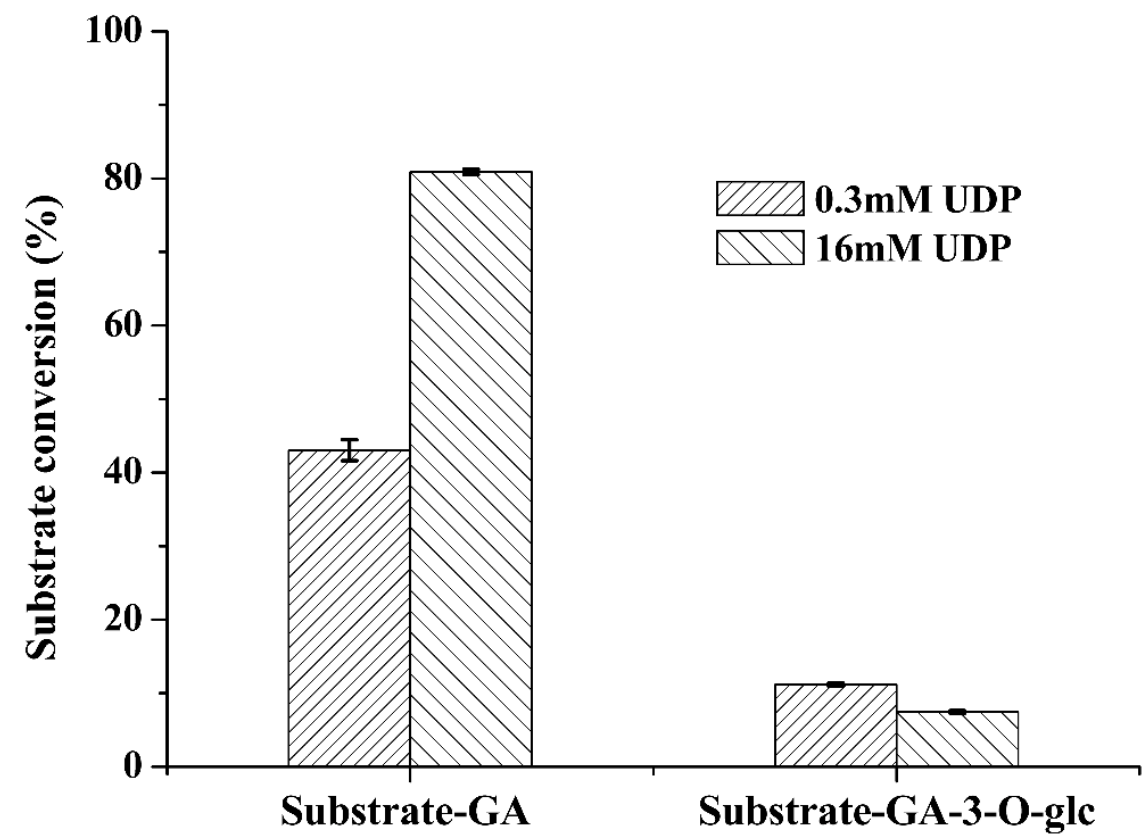

Figure S14. The inhibition of UDP on the first and second glycosylation of GA catalyzed by UGT73C11. 

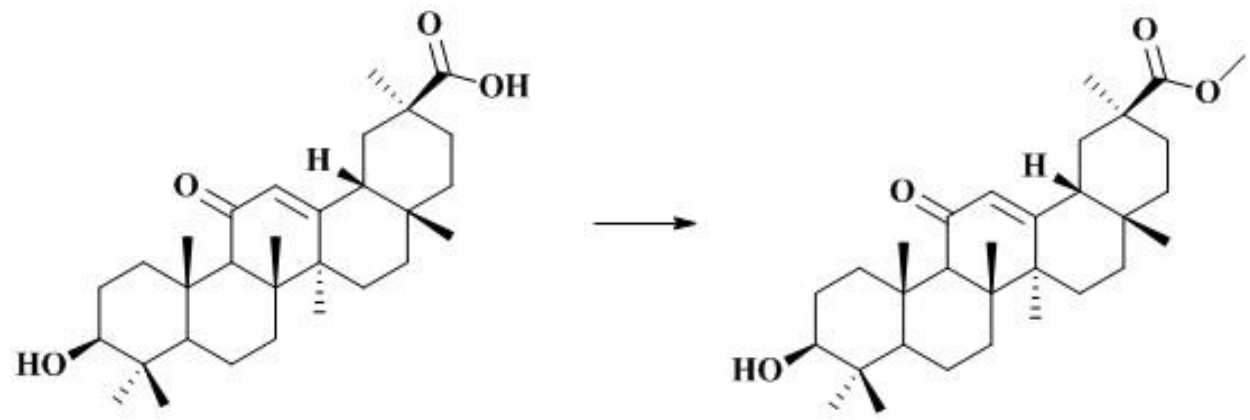

Figure S15. The synthesis of GA methyl ester from GA. $\mathrm{K}_{2} \mathrm{CO}_{3}(0.161 \mathrm{~g}, 1.168 \mathrm{mmol})$ was added to a solution of GA $(0.500 \mathrm{~g}, 1.062 \mathrm{mmol})$ in DMF $(10 \mathrm{~mL})$. The mixture was stirred for $20 \mathrm{~min}$ at room temperature. Then $\mathrm{CH}_{3} \mathrm{I}(0.793 \mathrm{~mL}, 1.274 \mathrm{mmol})$ was added to the reaction mixture. After stirring for $0.5 \mathrm{~h}$, the reaction mixture was poured into $\mathrm{H}_{2} \mathrm{O}(20 \mathrm{~mL})$ and extracted with $\mathrm{CH}_{2} \mathrm{Cl}_{2}$. The organic layer was washed with water and brine, and evaporated under vacuum. The resulting oil was mixed with petroleum ether and GA methyl ester was precipitated as white solid $(0.492 \mathrm{~g}, 1.015 \mathrm{mmol}$, 95.57\%). HRMS (ESI) $(\mathrm{M}+\mathrm{H})^{+} \mathrm{m} / \mathrm{z}$ 485.3623, calcd for $\mathrm{C}_{31} \mathrm{H}_{49} \mathrm{O}_{4}$ 485.3625. ${ }^{1} \mathrm{H}$ NMR $\left(\mathrm{CDCl}_{3}, 400 \mathrm{MHz}\right) \delta: 5.66(\mathrm{~s}, 1 \mathrm{H}, \mathrm{H}-12), 3.69\left(\mathrm{~s}, 3 \mathrm{H}, \mathrm{OCH}_{3}\right), 3.23\left(\mathrm{dd}, J_{l}=10.8 \mathrm{~Hz}\right.$, $\left.J_{2}=5.6 \mathrm{~Hz}, 1 \mathrm{H}, \mathrm{H}-3\right), 2.80\left(\mathrm{dt}, J_{1}=13.5 \mathrm{~Hz}, J_{2}=3.6 \mathrm{~Hz}, 1 \mathrm{H}, \mathrm{H}-1 \mathrm{a}\right), 2.34(\mathrm{~s}, 1 \mathrm{H}, \mathrm{H}-$ 9), $2.08\left(\mathrm{dd}, J_{l}=14.1 \mathrm{~Hz}, J_{2}=3.6 \mathrm{~Hz}, 1 \mathrm{H}, \mathrm{H}-18\right), 2.04-1.96$ (m, $\left.2 \mathrm{H}, \mathrm{H}-15 \mathrm{a}, \mathrm{H}-21 \mathrm{a}\right)$, $1.92\left(\mathrm{dt}, J_{1}=13.6 \mathrm{~Hz}, J_{2}=4.3 \mathrm{~Hz}, 1 \mathrm{H}, \mathrm{H}-19 \mathrm{a}\right), 1.83\left(\mathrm{dt}, J_{1}=13.6 \mathrm{~Hz}, J_{2}=4.4 \mathrm{~Hz}, 1 \mathrm{H}\right.$, H-16a), 1.72-1.56 (m, 3 H, H-2a, 2b, H-7a), 1.52-1.38 (m, 5 H, H-19b, H-6a,6b, H-7b, H-22a), 1.37 (s, 3 H, 27-CH3), 1.34-1.16 (m, 3 H, H-21b, H-22b, H-16b), 1.15 (s, 3 H, 28- $\left.\mathrm{CH}_{3}\right), 1.14$ (s, $\left.3 \mathrm{H}, 25-\mathrm{CH}_{3}\right), 1.13$ (s, $\left.3 \mathrm{H}, 26-\mathrm{CH}_{3}\right), 1.06-1.02$ (m, $\left.1 \mathrm{H}, \mathrm{H}-15 \mathrm{~b}\right), 1.01$ (s, $\left.3 \mathrm{H}, 23-\mathrm{CH}_{3}\right), 0.99-0.94$ (m, $\left.1 \mathrm{H}, \mathrm{H}-1 \mathrm{~b}\right), 0.81$ (s, $\left.6 \mathrm{H}, 24-\mathrm{CH}_{3}, 29-\mathrm{CH}_{3}\right), 0.70$ (d, $J=$ $11.8 \mathrm{~Hz}, 1 \mathrm{H}, \mathrm{H}-5 \mathrm{~d})$. 


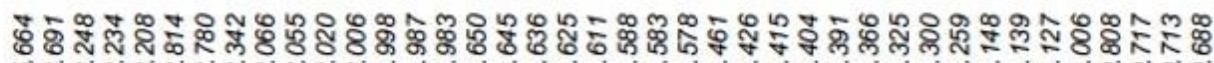
ద

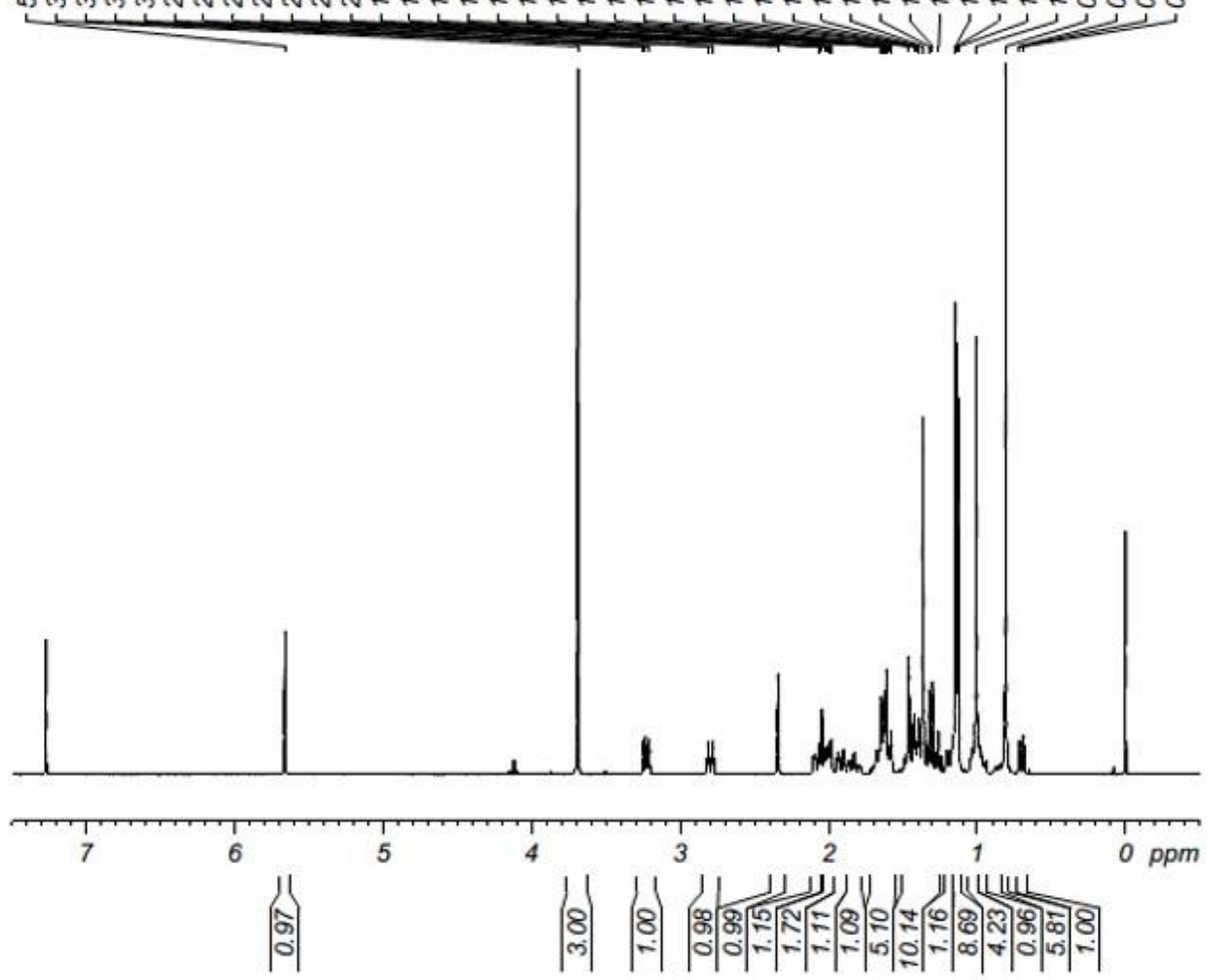

Figure S16. ${ }^{1} \mathrm{H}$ NMR spectrum of GA methyl ester. 


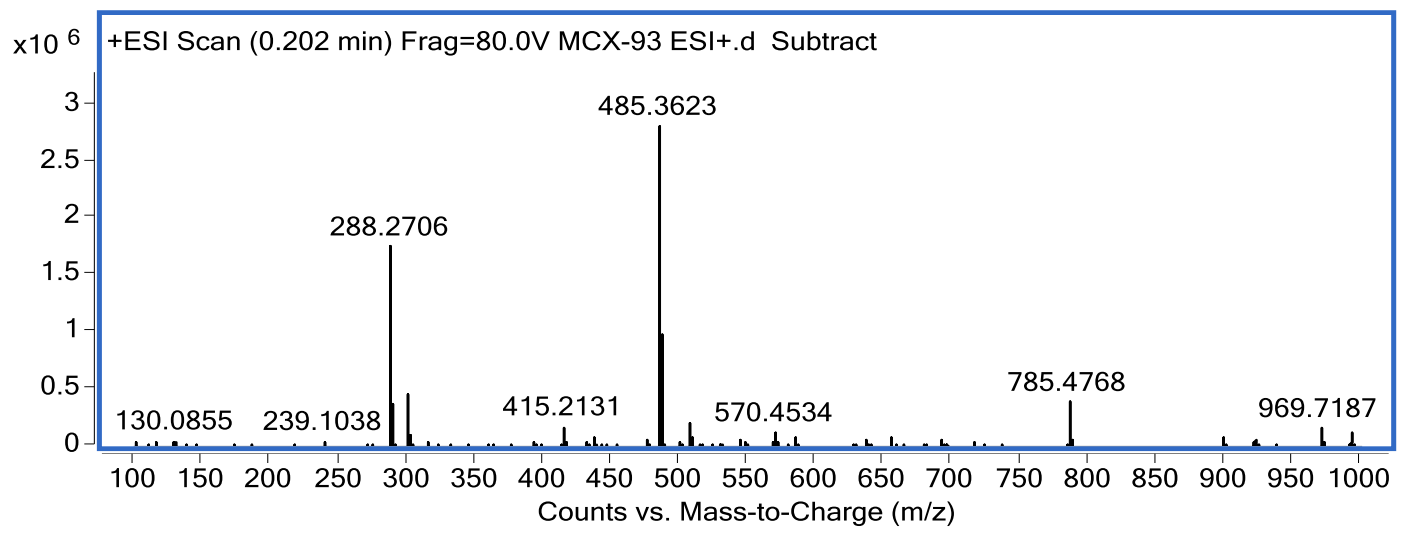

Figure S17. Mass spectrum of GA methyl ester 

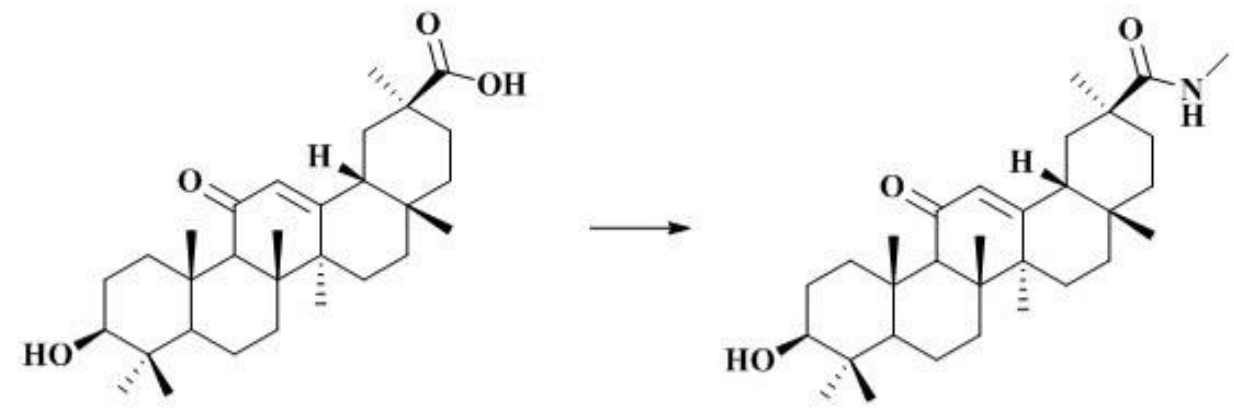

Figure S18. The synthesis of $\mathrm{N}-\mathrm{CH}_{3}-\mathrm{GA}$ amide from GA. GA $(0.300 \mathrm{~g}, 0.637 \mathrm{mmol})$ and $N, N^{\prime}$-carbonydiimiazole $(\mathrm{CDI})(0.124 \mathrm{~g}, 0.764 \mathrm{mmol})$ were suspended in anhydrous DMF $(2 \mathrm{~mL})$ and the mixture was stirred at $65^{\circ} \mathrm{C}$ for $2 \mathrm{~h}$. Then ice-cold methylamine (10 $\mathrm{mL} 40 \%$ aq) was slowly added to the mixture, which was stirred at room temperature overnight. The mixture was filtered and the cake was dried under vacuum. The cake was purified by column chromatography (silica gel, DCM/EtOH/ $\mathrm{NH}_{3} \cdot \mathrm{H}_{2} \mathrm{O}=$ 10:0.2:0.1) to give $\mathrm{N}-\mathrm{CH}_{3}$-GA amide $(0.103 \mathrm{~g}, 0.213 \mathrm{mmol}, 33.44 \%)$. HRMS (ESI) $(\mathrm{M}+\mathrm{H})^{+} \mathrm{m} / \mathrm{z} 484.3813$, calcd for $\mathrm{C}_{31} \mathrm{H}_{50} \mathrm{NO}_{3} 484.3785 .{ }^{1} \mathrm{H} \mathrm{NMR}\left(\mathrm{CDCl}_{3}, 400 \mathrm{MHz}\right) \delta$ : 5.67 (s, $2 \mathrm{H}, \mathrm{H}-12, \mathrm{NH}), 3.23\left(\mathrm{dd}, J_{1}=10.9 \mathrm{~Hz}, J_{2}=5.3 \mathrm{~Hz}, 1 \mathrm{H}, \mathrm{H}-3\right), 2.83$ (d, $J=4.5$ $\mathrm{Hz}, 1 \mathrm{H}, \mathrm{NH}-\mathrm{CH}_{3}$ ), 2.79 (dt, $\left.J_{1}=13.3 \mathrm{~Hz}, J_{2}=3.3 \mathrm{~Hz}, 1 \mathrm{H}, \mathrm{H}-1 \mathrm{a}\right), 2.34$ (s, $1 \mathrm{H}, \mathrm{H}-9$ ), $2.15\left(\mathrm{dd}, J_{l}=12.6 \mathrm{~Hz}, J_{2}=5.1 \mathrm{~Hz}, 1 \mathrm{H}, \mathrm{H}-18\right), 2.04\left(\mathrm{dt}, J_{1}=13.7 \mathrm{~Hz}, J_{2}=4.5 \mathrm{~Hz}, 1 \mathrm{H}\right.$, H-15a), 1.94 (d, $J=11.5 \mathrm{~Hz}, 1 \mathrm{H}, \mathrm{H}-21 \mathrm{a}), 1.84\left(\mathrm{dt}, J_{1}=13.7 \mathrm{~Hz}, J_{2}=4.7 \mathrm{~Hz}, 1 \mathrm{H}, \mathrm{H}-\right.$ 16a), 1.79-1.70 (m, 2 H, H-19a, 19b), 1.70-1.56 (m, 3 H, H-2a, 2b, H-7a), 1.53-1.38 (m, $6 \mathrm{H}, \mathrm{H}-6 \mathrm{a}, 6 \mathrm{~b}, \mathrm{H}-7 \mathrm{~b}, \mathrm{H}-22 \mathrm{a}, 22 \mathrm{~b}, \mathrm{H}-21 \mathrm{~b}), 1.37$ (s, $\left.3 \mathrm{H}, 27-\mathrm{CH}_{3}\right), 1.19$ (dd, $J_{1}=13.9 \mathrm{~Hz}$, $\left.J_{2}=2.3 \mathrm{~Hz}, 1 \mathrm{H}, \mathrm{H}-16 \mathrm{~b}\right), 1.13\left(\mathrm{~s}, 3 \mathrm{H}, 28-\mathrm{CH}_{3}\right), 1.12\left(\mathrm{~s}, 6 \mathrm{H}, 25-\mathrm{CH}_{3}, 26-\mathrm{CH}_{3}\right), 1.05-$ $1.01(\mathrm{~m}, 1 \mathrm{H}, \mathrm{H}-15 \mathrm{~b}), 1.01\left(\mathrm{~s}, 3 \mathrm{H}, 23-\mathrm{CH}_{3}\right), 0.96\left(\mathrm{dd}, J_{1}=13.0 \mathrm{~Hz}, J_{2}=4.6 \mathrm{~Hz}, 1 \mathrm{H}\right.$, $\mathrm{H}-1 \mathrm{~b}), 0.82\left(\mathrm{~s}, 6 \mathrm{H}, 24-\mathrm{CH}_{3}\right), 0.81\left(\mathrm{~s}, 6 \mathrm{H}, 29-\mathrm{CH}_{3}\right), 0.70$ (dd, $J_{1}=11.8 \mathrm{~Hz}, J_{2}=1.9 \mathrm{~Hz}$, $1 \mathrm{H}, \mathrm{H}-5 \mathrm{~d})$. 


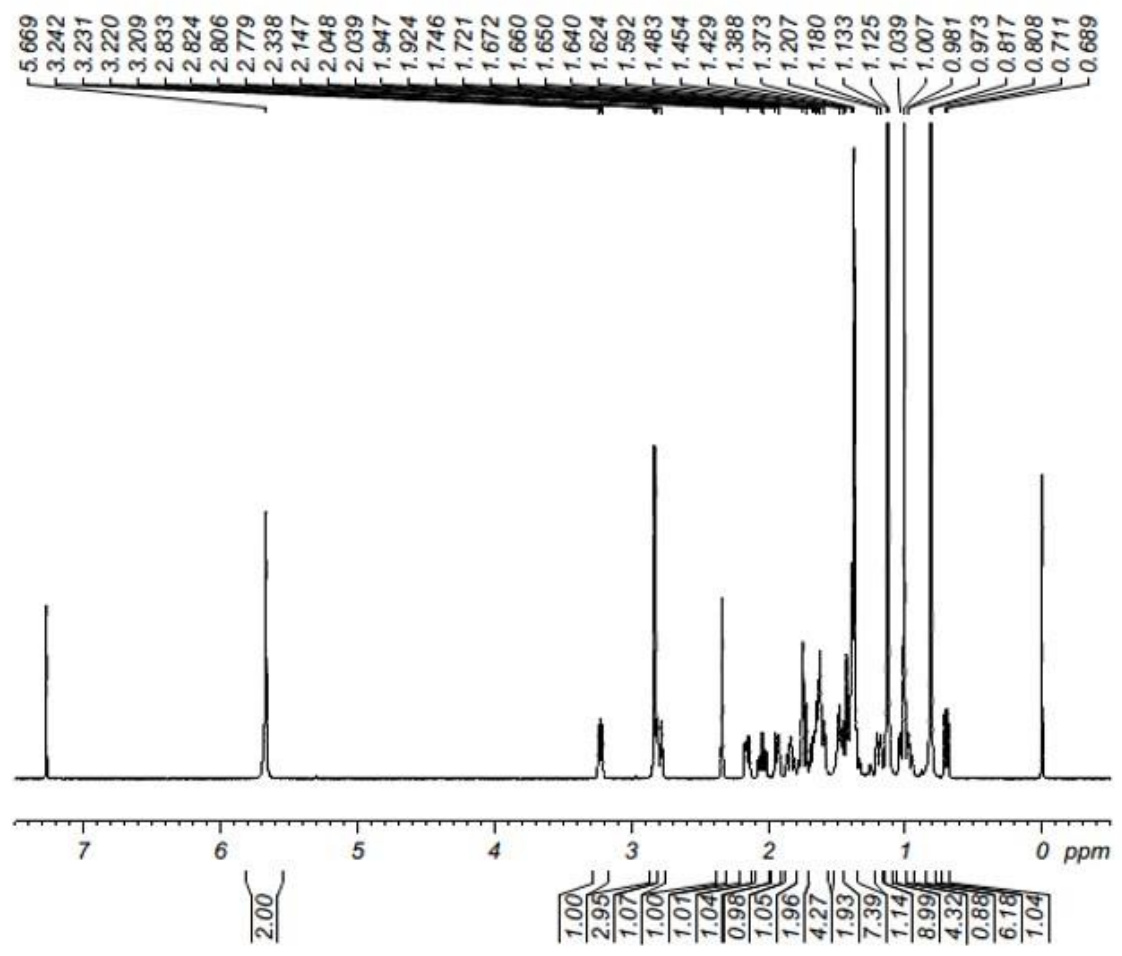

Figure S19. ${ }^{1} \mathrm{H}$ NMR spectrum of $\mathrm{N}-\mathrm{CH}_{3}-\mathrm{GA}$ amide. 


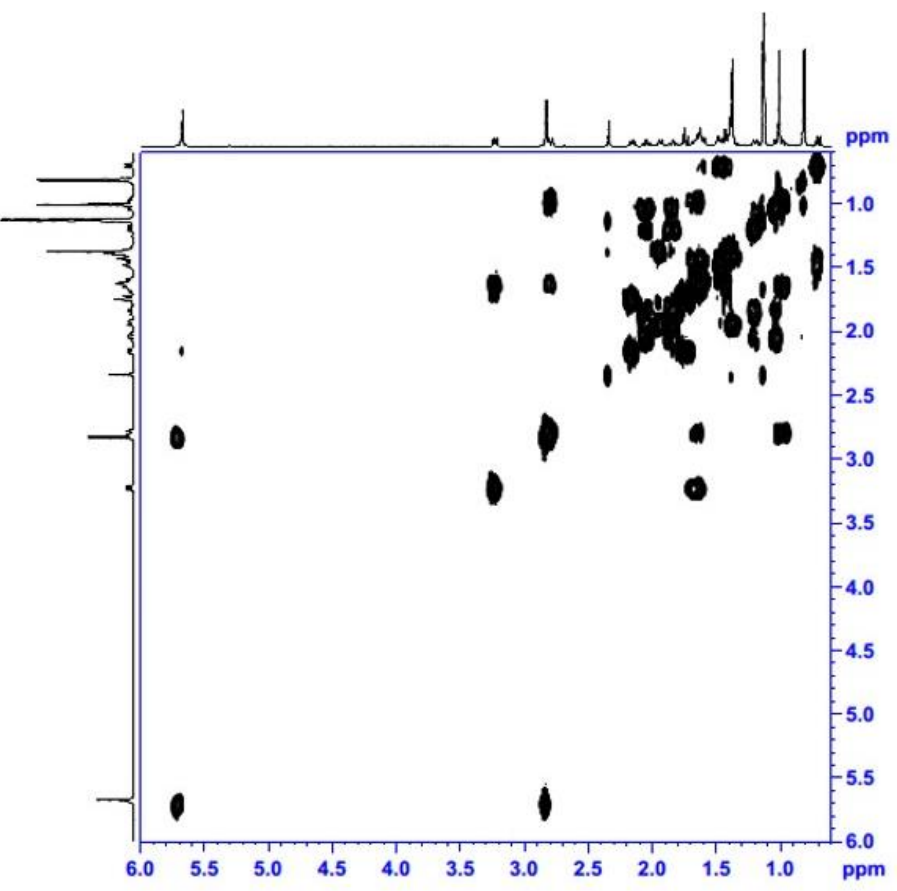

Figure S20. COSY spectrum of $\mathrm{N}-\mathrm{CH}_{3}-\mathrm{GA}$ amide. 


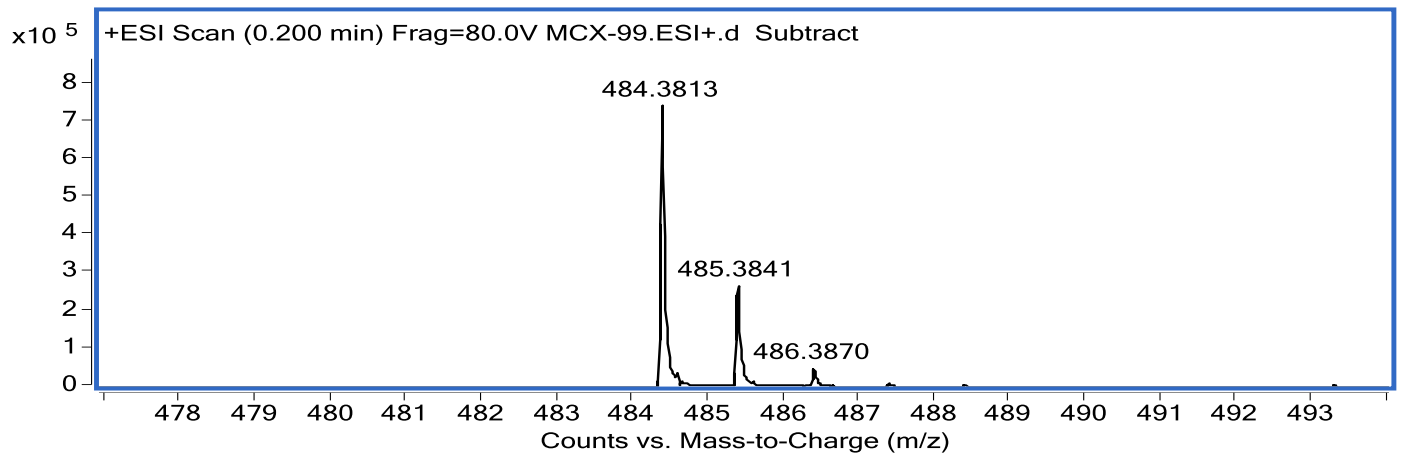

Figure S21. Mass spectrum of $\mathrm{N}-\mathrm{CH}_{3}-\mathrm{GA}$ amide. 
(a)
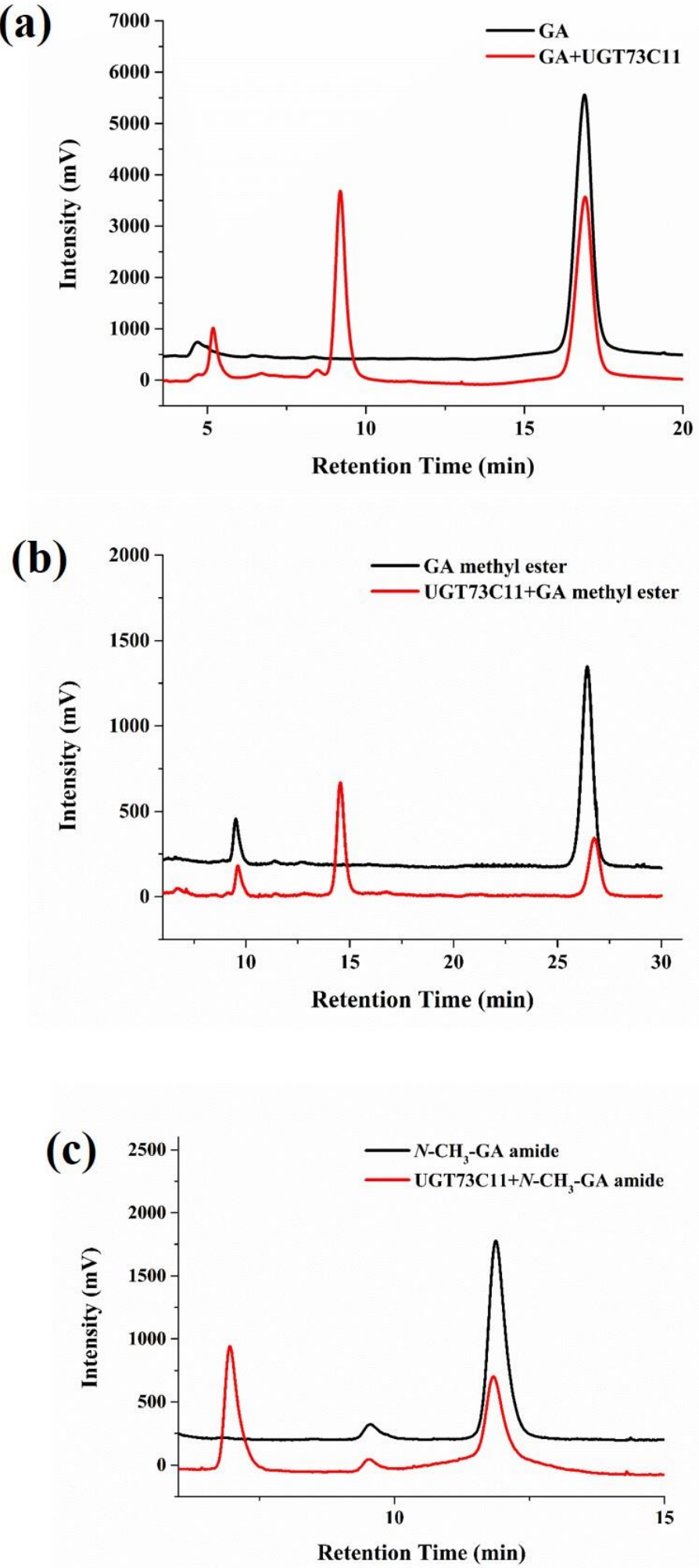

Figure S22. The HPLC chromatograph glycosylation of GA (a), GA methyl ester (b) and $\mathrm{N}-\mathrm{CH}_{3}-\mathrm{GA}$ amide (c) catalyzed by UGT3C11 with UDP recycling. 